\title{
Mast cells and neutrophils mediate peripheral motor pathway degeneration in ALS
}

\author{
Emiliano Trias, ${ }^{1}$ Peter H. King, ${ }^{2,3}$ Ying Si, ${ }^{2,3}$ Yuri Kwon, ${ }^{2}$ Valentina Varela, ${ }^{1}$ Sofía Ibarburu, ${ }^{1}$ \\ Mariángeles Kovacs, ${ }^{1}$ Ivan C. Moura, ${ }^{4,5,6,7,8,9}$ Joseph S. Beckman, ${ }^{10}$ Olivier Hermine, ${ }^{4,5,6,7,8,9,11,12,13}$ \\ and Luis Barbeito ${ }^{1}$
}

IInstitut Pasteur de Montevideo, Uruguay. ${ }^{2}$ Department of Neurology, University of Alabama, Birmingham, Alabama, USA. ${ }^{3}$ Birmingham Veterans Affairs Medical Center, Birmingham, Alabama, USA. ${ }^{4}$ Imagine Institute, Hôpital Necker, Paris, France. ${ }^{5}$ INSERM UMR 1163, Laboratory of Cellular and Molecular Mechanisms of Hematological Disorders and Therapeutic Implications, Paris, France. ${ }^{6}$ Paris Descartes-Sorbonne Paris Cité University, Imagine Institute, Paris, France. ${ }^{7} \mathrm{CNRS}$ ERL 8254, Paris, France. ${ }^{8}$ Laboratory of Excellence GR-Ex, Paris, France. ${ }^{9}$ Equipe Labélisée par la Ligue Nationale contre le cancer, Nantes, France. ${ }^{10}$ Linus Pauling Institute, Department of Biochemistry and Biophysics, Environmental Health Sciences Center, Oregon State University, Corvallis, Oregon, USA. "AB Science, Paris, France. ${ }^{12}$ Department of Hematology, Necker Hospital, Paris, France. ${ }^{13}$ Centre national de référence des mastocytoses (CEREMAST), Paris, France.

\begin{abstract}
Neuroinflammation is a recognized pathogenic mechanism underlying motor neuron degeneration in amyotrophic lateral sclerosis (ALS), but the inflammatory mechanisms influencing peripheral motor axon degeneration remain largely unknown. A recent report showed a pathogenic role for c-Kit-expressing mast cells mediating inflammation and neuromuscular junction denervation in muscles from SOD1 ${ }^{\text {C93A }}$ rats. Here, we have explored whether mast cells infiltrate skeletal muscles in autopsied muscles from ALS patients. We report that degranulating mast cells were abundant in the quadriceps muscles from ALS subjects but not in controls. Mast cells were associated with myofibers and motor endplates and, remarkably, interacted with neutrophils forming large extracellular traps. Mast cells and neutrophils were also abundant around motor axons in the extensor digitorum longus muscle, sciatic nerve, and ventral roots of symptomatic SOD1 ${ }^{\text {C93A }}$ rats, indicating that immune cell infiltration extends along the entire peripheral motor pathway. Postparalysis treatment of SOD1C93A rats with the tyrosine kinase inhibitor drug masitinib prevented mast cell and neutrophil infiltration, axonal pathology, secondary demyelination, and the loss of type $2 \mathrm{~B}$ myofibers, compared with vehicle-treated rats. These findings provide further evidence for a yet unrecognized contribution of immune cells in peripheral motor pathway degeneration that can be therapeutically targeted by tyrosine kinase inhibitors.
\end{abstract}

Conflict of interest: $\mathrm{OH}$ is a cofounder and shareholder of AB Science.

License: Copyright 2018, American Society for Clinical Investigation.

Submitted: June 29, 2018

Accepted: August 29, 2018

Published: October 4, 2018

Reference information: JCI Insight. 2018;3(19):e123249. https://doi.org/10.1172/jici. insight.123249.

\section{Introduction}

Motor neuron degeneration and distal motor axonopathy are among the best-recognized pathophysiological features of amyotrophic lateral sclerosis (ALS), a progressive and fatal neurodegenerative disease leading to muscle weakness and paralysis. Survival after diagnosis varies from 3 to 5 years (1), largely determined by the rate of spread of neuronal pathology in the central (CNS) and peripheral nervous systems (PNS). The etiology of ALS remains largely unknown and there is a poor understanding of the pathological mechanisms underlying the ineluctable progression of paralysis after disease onset. Evidence in ALS patients and murine models expressing the ALS-linked SOD1 mutations indicate that lower motor neuron degeneration starts peripherally by a distal axonopathy before overt loss of motor neuron cell bodies (2-4). Such dyingback motor neuropathy represents a yet elusive pathogenic process that could be targeted by therapeutic interventions aiming to slow the paralysis progression, turning this fatal disease into a chronic condition.

Degeneration of motor axons in ALS has been associated with defective axonal transport, mitochondrial function, and/or stabilization of neuromuscular junctions (NMJs) (5-9). However, the role of inflammatory mechanisms influencing peripheral motor axons remains only partially known in comparison with the understanding of inflammation involving glial and immune cells in the CNS. Monocytes and macrophages 
infiltrate peripheral nerves and skeletal muscle of ALS patients and transgenic rodents $(10,11)$; however, there is conflicting evidence as to whether macrophages are deleterious $(12,13)$ or protective (14) in ALS. Besides the complex role of monocytes/macrophages, little is known about the influence of other immune cells on peripheral motor axon degeneration in ALS patients.

Mast cells have recently been shown to directly interact with degenerating motor nerve endings and motor endplates in SOD1 ${ }^{\mathrm{G} 93 \mathrm{~A}}$ rat skeletal muscle, with their number and degranulation pattern correlating with paralysis progression (10). Pharmacological downregulation of mast cells using the selective c-Kit receptor inhibitor drug masitinib reduces the rate of NMJ denervation and motor deficits in the SOD $1^{\mathrm{G} 93 \mathrm{~A}}$ rats $(10)$, thereby indicating a role of mast cells in accelerating paralysis progression. Mast cells are granulated hematopoietic-derived immune cells that migrate and differentiate within tissues as precursors expressing the tyrosine kinase receptor c-Kit $(15,16)$. Mast cells serve as sentinel cells in tissues and can be activated upon damage, orchestrating complex inflammatory responses through the release of mediators and enzymes $(16,17)$. They also interact with sensory and autonomic nerve terminals to mediate neurogenic inflammation (18). However, the mechanism by which mast cells in concert with other immune effectors influence the motor nerve terminals in ALS remains unknown. Mast cells have the ability to increase the vascular permeability at the inflamed site, initiating the recruitment, clustering, and activation of neutrophils (19-21), with the potential to damage neuromuscular cellular components.

Neutrophils are polymorphonuclear leukocytes generated in the bone marrow from myeloid precursors (22). These cells have recently been implicated as potent cytotoxic effectors in neurodegenerative pathologies such as Alzheimer's disease (23-25). In skeletal muscle, neutrophils are recognized by their role in mediating myofiber damage and atrophy in physiological or pathological conditions (26, 27). Neutrophils have the ability to kill pathogens and also influence local cells and immune responses through the secretion of granules formed during their maturation containing cytotoxic and proinflammatory proteins such as myeloperoxidase (MPO), lactoferrin, metalloproteases, and elastase (28, 29). Highly activated neutrophils can form neutrophil extracellular traps (NETs) composed of DNA fibers with attached chromatin components, MPO, and elastase $(29,30)$. However, the association of neutrophils with motor nerve terminals in ALS degenerating muscles has not been explored in the context of motor neuron disease.

In this scenario, it remains unknown whether mast cells in association with neutrophils exert a pathogenic effect in ALS patients. It is also unknown whether mast cell- and neutrophil-driven pathology extends along the peripheral motor pathways during paralysis progression in the SOD $1{ }^{\mathrm{G} 93 \mathrm{~A}}$ rat model. Such knowledge would be relevant for the development of biomarkers and new therapeutic agents that could downregulate peripheral immune cell-mediated inflammation.

The tyrosine kinase inhibitor drug masitinib has been shown to prolong postparalysis survival by delaying motor neuron death and NMJ denervation in SOD $1^{\mathrm{G} 93 \mathrm{~A}}$ rats $(10,31)$. Masitinib is a multifaceted drug that selectively inhibits type-3 tyrosine kinase receptors, including c-Kit and colony-stimulating factor 1 receptor (CSF-1R) (32, 33). By targeting CSF-1R and c-Kit, masitinib can prevent excessive aberrant microgliosis in the spinal cord and accumulation of mast cells in the skeletal muscles of symptomatic SOD $1^{\mathrm{G} 93 \mathrm{~A}}$ rats, respectively $(10,31)$. Masitinib has been clinically tested in a phase 3 ALS trial, demonstrating a significant therapeutic benefit for its primary endpoint (34). Thus, masitinib can be used in SOD $1^{\mathrm{G} 93 \mathrm{~A}}$ rats as a pharmacological tool to downregulate mast cells, as well as subsequent mast cell-mediated inflammatory events influencing the degeneration of the peripheral motor pathway.

We investigated for the occurrence of mast cells and neutrophils in autopsied quadriceps femoris muscles from subjects with sporadic ALS as compared with muscle from control donors. To further understand the behavior of these cells during paralysis progression we analyzed the extensor digitorum longus (EDL) muscle, sciatic nerve, and ventral roots of SOD $1^{\mathrm{G} 93 \mathrm{~A}}$ rats. We also explored whether tyrosine kinase inhibition with masitinib in $\mathrm{SOD} 1^{\mathrm{G} 93 \mathrm{~A}}$ rats ameliorates the paralysis progression by modifying mast cell and neutrophil responses.

\section{Results}

Infiltration and degranulation of mast cells into the skeletal muscle of autopsied ALS subjects. Based on previous findings in affected muscles from SOD $1^{\mathrm{G} 93 \mathrm{~A}}$ rats during paralysis progression (10), we tested the hypothesis that mast cells accumulate in significant numbers in autopsied quadriceps muscles from ALS subjects. 
Table 1. Clinical characteristics of ALS and control subjects included in the study

\begin{tabular}{|c|c|c|c|c|c|c|c|c|}
\hline Subject & Muscle & MRC grade A $^{A}$ & Age (years) & Sex & Disease onset & $\begin{array}{c}\text { Survival } \\
\text { (Months) }\end{array}$ & $\begin{array}{l}\text { Postmortem } \\
\text { tissue } \\
\text { processing } \\
\text { (hours) }\end{array}$ & $\begin{array}{c}\text { EMG }^{\mathrm{B}} \\
\text { denervation }\end{array}$ \\
\hline ALS 1 & vastus & 3 & 63 & $M$ & Leg & 44 & 7.0 & $\begin{array}{c}\text { Active and } \\
\text { chronic }\end{array}$ \\
\hline ALS 2 & vastus & 1 & 69 & $\mathrm{~F}$ & Leg & 50 & 3.0 & Active \\
\hline ALS 3 & vastus & 3 & 64 & $M$ & Leg & 35 & 6.5 & Chronic \\
\hline ALS 4 & vastus & $3^{c}$ & 59 & $\mathrm{~F}$ & Arm & 26 & 13.0 & Active \\
\hline ALS 5 & vastus & 3 & 75 & $M$ & Bulbar & 55 & 4.3 & $\begin{array}{c}\text { Active and } \\
\text { chronic }\end{array}$ \\
\hline Control 1 & vastus & - & 61 & $M$ & - & - & 10.0 & - \\
\hline Control 2 & vastus & - & 68 & $M$ & - & - & 19 & - \\
\hline Control 3 & vastus & - & 59 & $M$ & - & - & 9.5 & - \\
\hline Control 4 & vastus & - & 90 & $M$ & - & - & 4.5 & - \\
\hline Control 5 & vastus & - & 62 & $M$ & - & - & 3.0 & - \\
\hline
\end{tabular}

${ }^{A}$ Medical Research Council (MRC) muscle power grade at clinic visit prior to death. ${ }^{\mathrm{B} E M C}$ performed at time of ALS diagnosis. ${ }^{\mathrm{C} M u s c l e}$ showed predominant spasticity.

Table 1 shows the characteristics of 5 ALS and 5 control subjects analyzed as well as the time of postmortem tissue processing. At the time of ALS diagnosis, there was electrophysiological evidence of quadriceps denervation in each patient. The clinical grade of the quadriceps muscle at the last clinic visit prior to death was similar in 4 of the 5 patients, with a score of 3 using the Medical Research Council (MRC) grading system. One of the patients, ALS 4, had severe spasticity in the quadriceps, reflecting predominant upper motor neuron dysfunction. Another patient, ALS 2, had end-stage paralysis of the quadriceps, as indicated by an MRC score of 1 . For histological characterization of the muscle samples, toluidine blue staining was used to identify metachromatic mast cells. As shown in Figure 1A and Supplemental Figure 1 (supplemental material available online with this article; https://doi.org/10.1172/jci.insight.123249DS1), the histoarchitecture of quadriceps muscle (vastus lateralis) from ALS subjects systemically displayed significant pathology including a large number of atrophic and disorganized muscle fibers, as well as increased density of nuclear clumps between myofibers. In control quadriceps muscles, mast cells were scarce, of small size, and nondegranulating, being mainly located at the perivascular regions (Figure 1A). In comparison, ALS quadriceps showed a systematic, albeit variable, and statistically significant increase of metachromatic mast cell density (Figure 1A and Supplemental Figure 1B). A comparative statistical analysis of mast cell numbers among patients and controls is shown in Supplemental Table 1. In addition, mast cells in ALS muscles displayed increased size, and frequent images of explosive degranulation that extended up to $50 \mu \mathrm{m}$ from the cell body (Figure 1A and Supplemental Figure 1B). Immunostaining for a mast cell-specific protease, chymase, further confirmed mast cell infiltration in ALS-affected muscles and the abundance of granules invading the neighboring myofibers (Figure 1, B and C, and Supplemental Figure 1B). Mast cells expressed c-Kit, a tyrosine kinase receptor found in mast cell precursors and during early differentiation (Figure 1D). In summary, these findings are in accordance with a previous report in symptomatic rats (10) and indicate that mast cells are involved in chronic inflammatory muscle response in ALS.

Mast cells interact with neutrophils and motor endplates in the skeletal muscle of ALS subjects. Mast cells have the ability to recruit and activate other immune cells through degranulation and release of inflammatory mediators $(19,21)$, with macrophages and T cells being previously described to infiltrate ALS-affected muscles $(11,13$, 35). However, it remains unknown whether mast cells associate with neutrophils in ALS muscles, particularly at the neuromuscular compartment. As shown in Figure 2 and Supplemental Figure 2, we used elastase and MPO immunohistochemistry to examine neutrophil infiltration. In quadriceps muscles from control subjects, neutrophils were absent in the endomysium but occasionally found associated with blood vessels. In contrast, endomysial neutrophils expressing elastase were numerous in ALS patients establishing direct contact with atrophic myofibers and forming multicellular clusters (Figure 2A and Supplemental Figure 2). Neutrophil number 
A

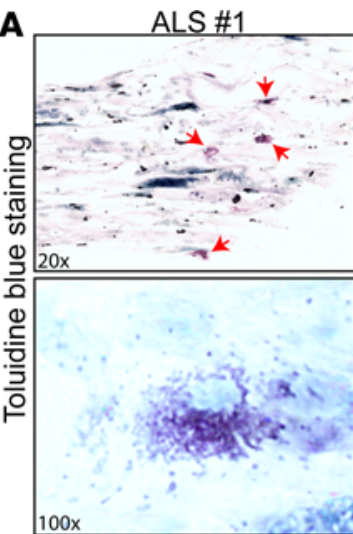

B
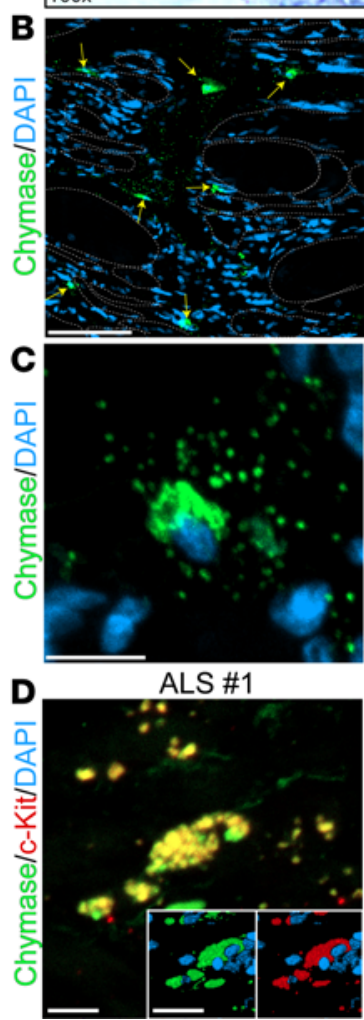
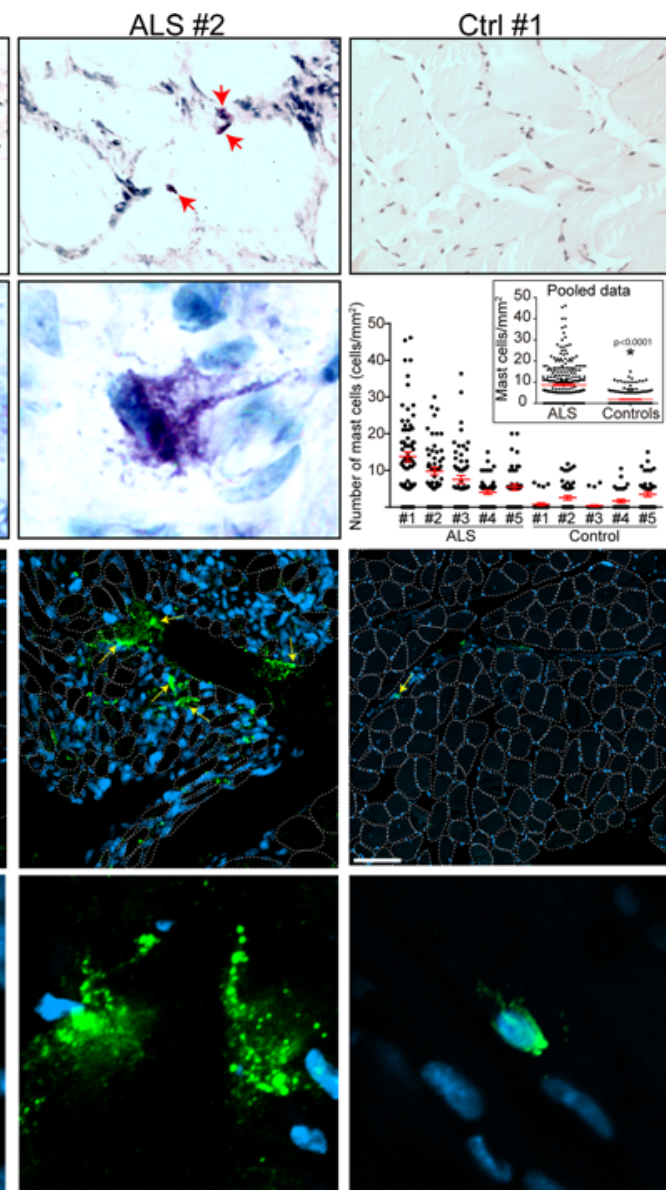

ALS \#2

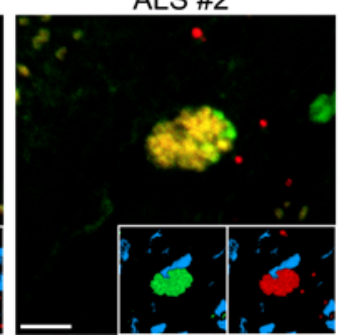

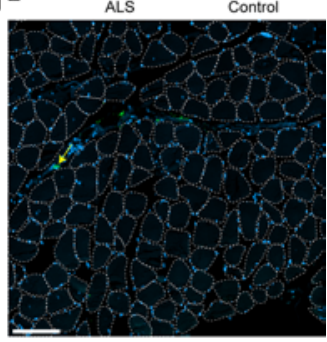

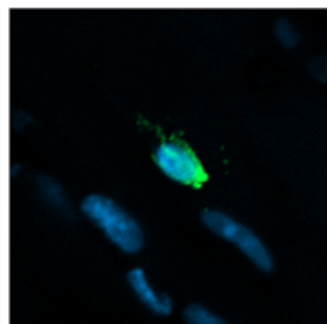

ALS \#3

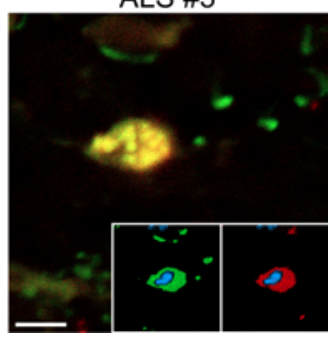

Figure 1. Mast cells infiltrate into and degranulate in skeletal muscle of ALS patients. (A) Representative images of toluidine blue staining showing the infiltration of mast cells into quadriceps muscles of ALS patients. Upper panels are low-magnification microphotographs showing the significant infiltration of mast cells (arrows) into degenerating muscle of ALS patients compared with controls, where few mast cells are observed and mostly associated with blood vessels. Note the significant infiltration and degranulation state of mast cells in ALS conditions (lower panels). The graph shows the quantitative analysis of mast cells into the quadriceps muscles of 5 ALS patients and 5 controls stained with toluidine blue. The graph inset shows the quantitative analysis of mast cell infiltration for pooled ALS patients compared with pooled controls. Data were analyzed by Kruskal-Wallis followed by Dunn's multiple-comparisons test (graph) and Mann-Whitney test, 2-tailed (graph inset), and expressed as mean $\pm \mathrm{SEM} ; P<0.05$ was considered statistically significant. (B) Representative confocal tile reconstruction showing chymase-positive mast cells (green, arrows) infiltrating quadriceps muscles from ALS and control donors. A marked infiltration of mast cells is seen in ALS patients when compared with controls, where few smaller chymase-positive mast cells are observed associated mostly with blood vessels. Dotted lines delimit myofibers. Scale bars: $100 \mu \mathrm{m}$. (C) Representative confocal microphotographs showing an important number of chymase-positive cells that show an irregular shape corresponding to a degranulating state, which is not observed in any of the controls analyzed. Scale bar: $10 \mu \mathrm{m}$. (D) Confocal microphotographs show the coexpression of the tyrosine kinase receptor c-Kit (red) and chymase (green) in a subpopulation of cells that resemble mast cell progenitors that infiltrate the muscle. Insets show $3 \mathrm{D}$ confocal reconstruction of separated channels. Scale bars: $5 \mu \mathrm{m}$ (colocalization) and $10 \mu \mathrm{m}$ (insets).

was significantly increased in ALS patients as compared with controls (graph in Figure 2A) and correlated with mast cell number $\left(R^{2}=0.78\right.$, graph in Supplemental Figure 2). A comparative statistical analysis of neutrophil number among patients and controls is shown in Supplemental Table 2. Immunoreactivity to MPO was also found in a subset of small-sized neutrophils occurring in ALS muscles but not in controls (Figure 2B). Notably, neutrophils in ALS muscles frequently formed NETs (Figure 2C), as demonstrated by extracellular mesh-like structures of DNA associated with extracellular elastase (Figure 2C). NET formation denotes full activation and subsequent death of neutrophils as well as high cytotoxic potential to surrounding cells. In addition, neutrophils and mast cells were frequently found in close contact with the NETs and with motor endplates labeled with $\alpha$-bungarotoxin (Figure 2, C-F), suggesting a concerted activity of both immune cells on the NMJ elements.

Neutrophil infiltration into the EDL muscle of SOD1 ${ }^{G 93 A}$ rats during paralysis progression. We tested the dynamics of neutrophil infiltration during paralysis progression in the neuromuscular compartment of SOD $1^{\mathrm{G} 93 \mathrm{~A}}$ rats as compared with previous data on mast cells (10). We hypothesized that infiltrating mast cells have the ability to recruit neutrophils, which in turn act as cytotoxic effectors. As shown in Figure 3, the number of elastase-positive neutrophils in the EDL muscle of nontransgenic (NonTg) rats was scarce and absent in the surroundings of motor endplates. At paralysis onset, neutrophils were infrequently associated with NMJs. In contrast, a sharp increase in neutrophil number was found 15 days after paralysis onset, with the cells being enlarged in size, forming clusters and NETs (Figure 3, A and B). Neutrophils were occasionally found in close contact 

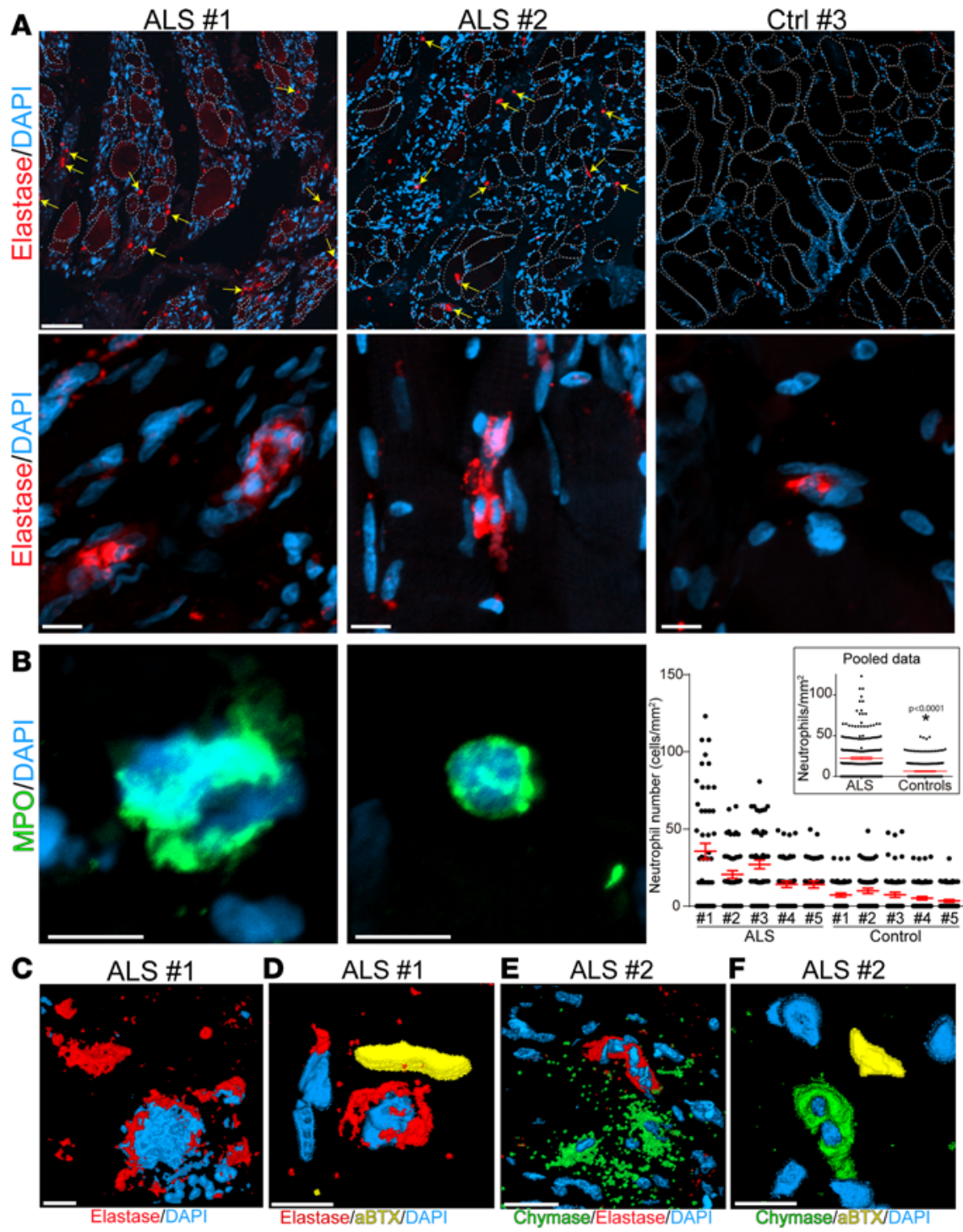

F ALS \#2

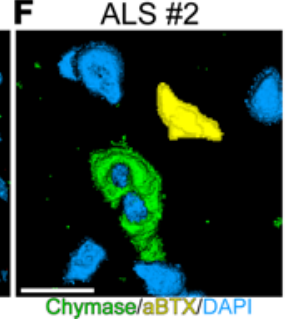

Figure 2. Neutrophils infiltrate into the degenerating skeletal muscle of ALS patients and interact with mast cells and NMJs. (A) Representative confocal tile reconstruction showing the infiltration of elastase-positive (red) neutrophils into 2 postmortem quadriceps samples from ALS patients and 1 control donor (upper panels). High magnification in panels of the second row show how neutrophils from ALS patients form aggregates resembling neutrophil extracellular traps. Neutrophils from control donors were seen associated with blood vessels and constitute small single cells that do not aggregate. Dotted lines delimit myofibers. Scale bars: $100 \mu \mathrm{m}$ in low-magnification panels, $10 \mu \mathrm{m}$ in high-magnification panels. (B) Representative high-magnification confocal images showing the infiltration of myeloperoxidase (MPO, green) into the degenerating quadriceps ALS muscles. Few or no MPO-positive cells are observed in control donors. Scale bars: $10 \mu \mathrm{m}$. The graph to the right shows the quantitative analysis of total elastase-positive neutrophils present in 10 - $\mu \mathrm{m}$ sections of quadriceps muscle for each ALS patient and control. The graph inset shows the quantitative analysis of elastase-positive neutrophil infiltration for pooled ALS patients compared with pooled controls. Data were analyzed by Kruskal-Wallis followed by Dunn's multiple-comparisons test (graph) and Mann-Whitney test, 2-tailed (graph inset) and are expressed as mean $\pm \mathrm{SEM} ; P<0.05$ was considered statistically significant. (C) Representative confocal surface 3D reconstruction showing neutrophil extracellular traps in quadriceps of an ALS case. Scale bar: $20 \mu \mathrm{m}$. (D) Representative 3D confocal reconstruction showing the interaction of elastase-positive neutrophils (red) with NMJs motor endplates (yellow). Scale bar: $10 \mu \mathrm{m}$. (E) Representative 3D confocal surface reconstruction showing the interaction of elastase-positive neutrophils (red) with degranulating chymase-positive mast cells (green). No degranulating mast cells or neutrophil-mast cell interactions are observed in control donors. Scale bar: $20 \mu \mathrm{m}$. (F) Representative confocal microphotograph showing the proximity of chymase-positive mast cells (green) to motor endplates (yellow) in the quadriceps muscle of an ALS patient. Scale bar: $10 \mu \mathrm{m}$.

with denervated NMJs and motor nerve terminals, with frequent images of cells engulfing neurofilaments, suggesting active axonal degradation (Figure 3B). A subpopulation of neutrophils expressing MPO also interacted with motor endplates and axonal terminals in the EDL muscle during advanced paralysis (Figure 3C).

Mast cell and neutrophil infiltration into the sciatic nerve and ventral roots of SOD1G93A rats. The association of mast cells and neutrophils with diseased motor nerve terminals in EDL muscles suggests they could also be involved in the dying-back of motor axons along spinal nerves. Thus, we examined mast cell phenotypic 


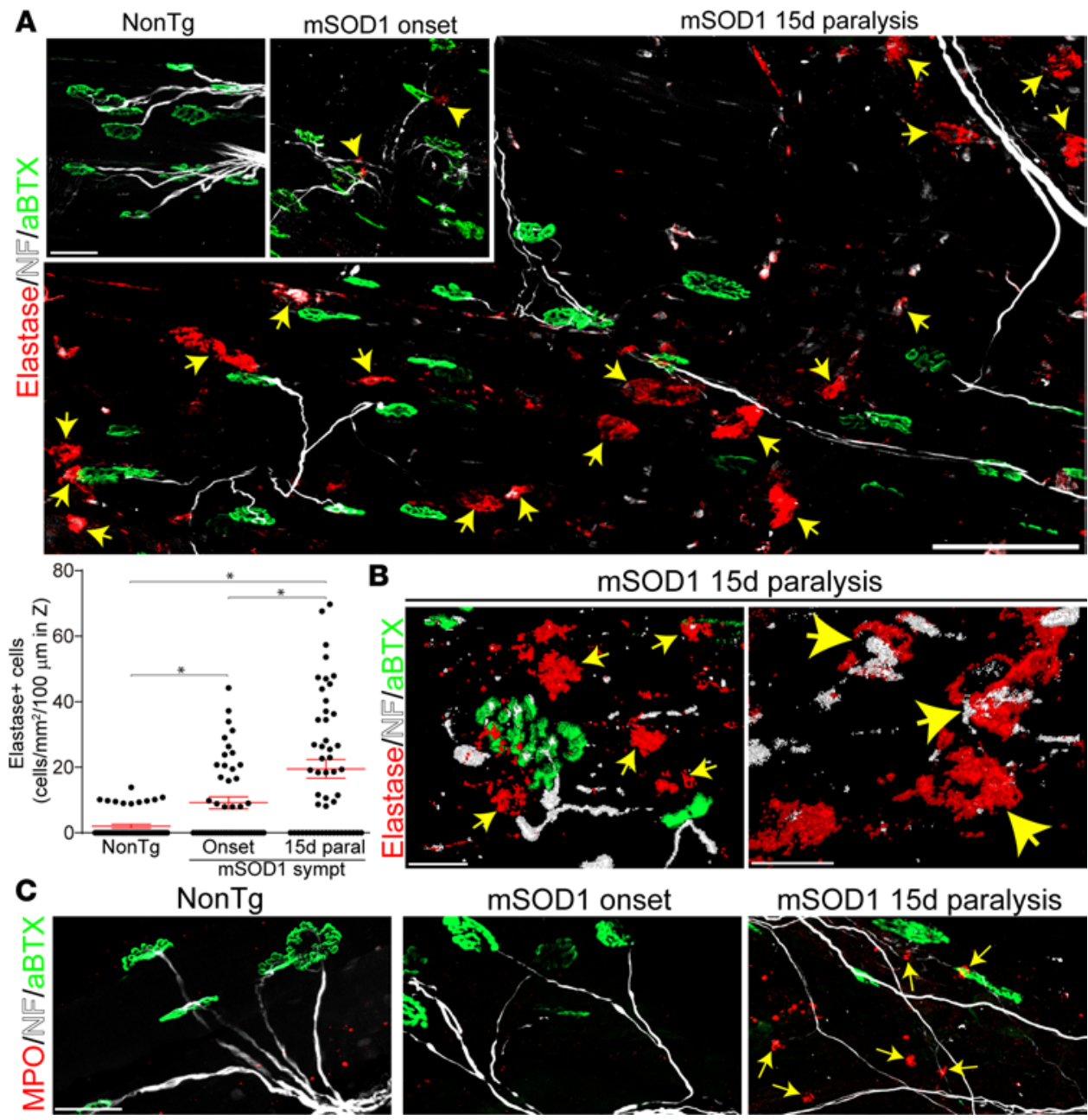

Figure 3. Neutrophils infiltrate NMJs of EDL degenerating muscles in mSOD1 symptomatic rats. Rat EDL muscle (whole mount) from SOD1 ${ }^{993 \mathrm{~A}}$ (onset and 15 days paralysis) and nontransgenic (NonTg) control were analyzed. (A) Representative confocal microphotograph showing elastase-positive neutrophil (red, arrows) infiltrating the surroundings of NMJs labeled with $\alpha$-bungarotoxin (green) and neurofilament (white) staining. Few elastase-positive cells were observed in NonTg (upper insets). Note the significant increase of elastase-positive neutrophils correlating with disease progression in the quantitative analysis showed in the graph below. Data were analyzed by Kruskal-Wallis followed by Dunn's multiple-comparisons test and are expressed as the mean \pm SEM; ${ }^{*} P<0.05$. Scale bars: $50 \mu \mathrm{m}$ in upper insets, $100 \mu \mathrm{m}$ in mSOD1 15-day paralysis. (B) Confocal 3D reconstruction showing the proximity of elastase-positive neutrophils (red, arrows) to NMJs (green/white, left panel). The panel to the right shows a representative confocal 3D reconstruction that shows elastase-positive neutrophils (red, arrows) phagocytizing neurofilament (white) debris. Scale bars: $20 \mu \mathrm{m}$. (C) Myeloperoxidase (MPO) antibody was also used to characterize the infiltration of MPO-positive neutrophils (red, arrows) into the degenerating EDL muscle of mSOD1 symptomatic rats. Note the increased number of MPO-positive cells that correlate with paralysis progression. Few or no neutrophils are observed in NonTg animals. Scale bar: $50 \mu \mathrm{m}$.

features in histological sections of the SOD $1^{\mathrm{G} 93 \mathrm{~A}}$ rat sciatic nerves and ventral roots during paralysis progression (Figure 4). In healthy sciatic nerves from NonTg rats, mast cells were rarely found in the endoneural space but occurred in low numbers around blood vessels at the perineurium and epineurium. In contrast, mast cells were abundant in symptomatic rats, aligned on the apparent trajectory of degenerating axons, with the number sharply increasing after paralysis onset (Figure 4, A and B). Mast cells displayed increased size and abundant metachromatic granules when stained with toluidine blue, with frequent occurrence of explosive degranulation (Figure 4A, inset). The density of mast cells was significantly increased at paralysis onset and advanced paralysis, compared with age-matched NonTg healthy controls (Figure 4C). Notably, the density of degranulating mast cells, a more relevant measure of mast cell activity, was augmented by 2-fold and 5-fold at paralysis onset and advanced paralysis, respectively, as compared with NonTg animals (Figure 4C). Immunostaining for tryptase further confirmed the progressive mast cell infiltration into the sciatic nerve but also a great abundance of tryptase-positive granules invading the neighboring endoneurium (Figure 4, B and C). In comparison, no significant mast cell infiltration was observed in either the spinal cord or the dorsal root ganglia in any stage of the disease (Supplemental Figure 3). 


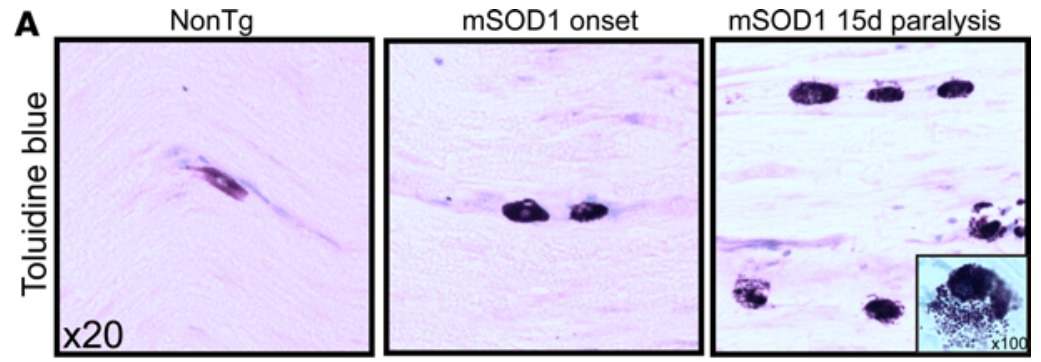

B
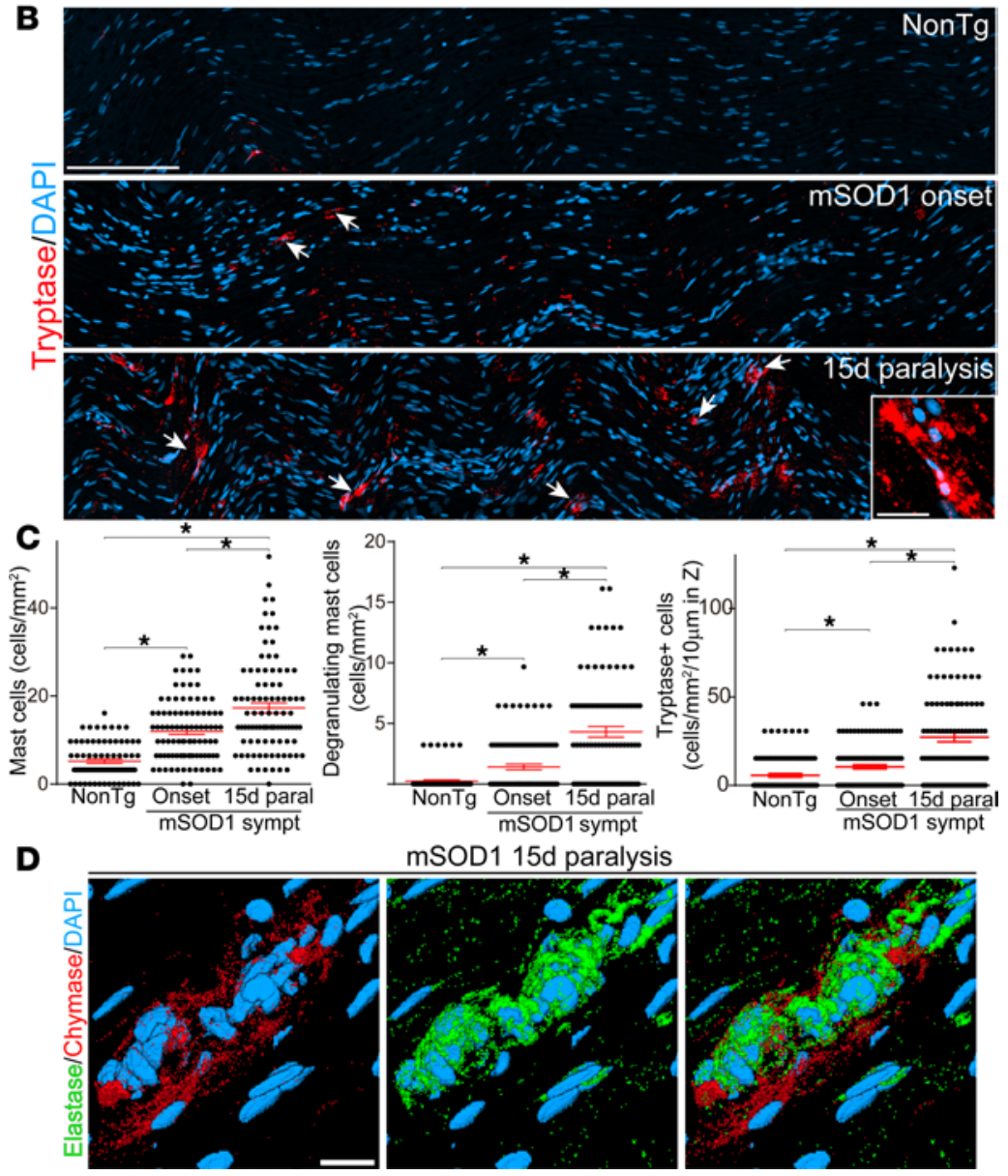

Figure 4. Infiltration and degranulation of mast cells into sciatic nerve endoneurium after paralysis onset in mSOD1 rats. Longitudinal $10-\mu \mathrm{m}$ cryostat sciatic nerve sections of nontransgenic (NonTg) and mSOD1 (onset and 15-day paralysis) rats. (A) Using toluidine blue staining, few endoneurial mast cells displaying metachromasia were observed in NonTg and mSOD1-onset sciatic nerve as compared with numerous mast cells 15 days after paralysis onset. High-magnification image ( $\times 100$, inset) shows degranulating mast cells, which are commonly observed in mSOD1-advanced when compared with NonTg and mSOD1-onset, where few degranulating mast cells are observed. (B) Representative confocal images comparing the infiltration of tryptase-positive mast cells (red, arrows) among conditions. Few mast cells are observed in NonTg and mSOD1-onset conditions when compared with mSOD1 $15 \mathrm{~d}$ paralysis. Inset in lowest panel shows degranulating tryptase-positive mast cells (red). Scale bars: $100 \mu \mathrm{m}$ in lower magnification panels, $20 \mu \mathrm{m}$ in insets. (C) Quantitative analysis of total and degranulating mast cell number, toluidine blue staining, and tryptase staining. Note the sharp increase in mast cells between mSOD1 onset and 15 -day paralysis. All quantitative data are expressed as the mean \pm SEM ( $n=4$ animals/condition). Data were analyzed by Kruskal-Wallis followed by Dunn's multiple-comparisons test; ${ }^{*} P<0.05$. (D) $3 D$ representative reconstruction of mast cell/neutrophil aggregates observed in the sciatic nerve 15 days after paralysis onset. Note chymase-positive mast cells (red) organizing at the surface of elastase-positive neutrophil aggregates (green) that aligned in the endoneurium. Scale bar: $10 \mu \mathrm{m}$.

Notably, a subpopulation of mast cells stained for chymase was found in direct contact with neutrophil clusters aligned along degenerating axons, indicating a direct interaction between these cells (Figure 4D). Such heterotypic aggregates were typically formed by several compacted neutrophils surrounded by mast cells expressing chymase, a protease with recognized chemoattractant ability for neutrophils $(20,36)$.

Neutrophil aggregates were a remarkable feature of diseased sciatic nerve during postparalysis pathology (Figure 5); the estimated cell number increased by 3.5 -fold between paralysis onset and advanced paralysis (Figure 5A). Neutrophils were frequently associated with myelin debris and displayed myelin phagocytosis (Figure 5B), which is in agreement with their role in myelin clearance in Wallerian degeneration (37). NET formation with typical DNA mesh-like structures was frequently observed in the degenerating sciatic nerve (Figure 5B). Neutrophil aggregates were also observed in the ventral roots of SOD $1^{\mathrm{G} 93 \mathrm{~A}}$ rats at 15 days after paralysis onset (Figure 5C), further indicating that these cells are closely associated with axonal degeneration.

Masitinib reduces mast cell and neutrophil accumulation and motor pathway degeneration. Masitinib potently inhibits the c-Kit-mediated pathway, inhibiting mast cell migration and activation $(32,33)$. It also inhibits CSF$1 \mathrm{R}$ associated with macrophage proliferation and activation (31). To determine the pathogenic contribution of 


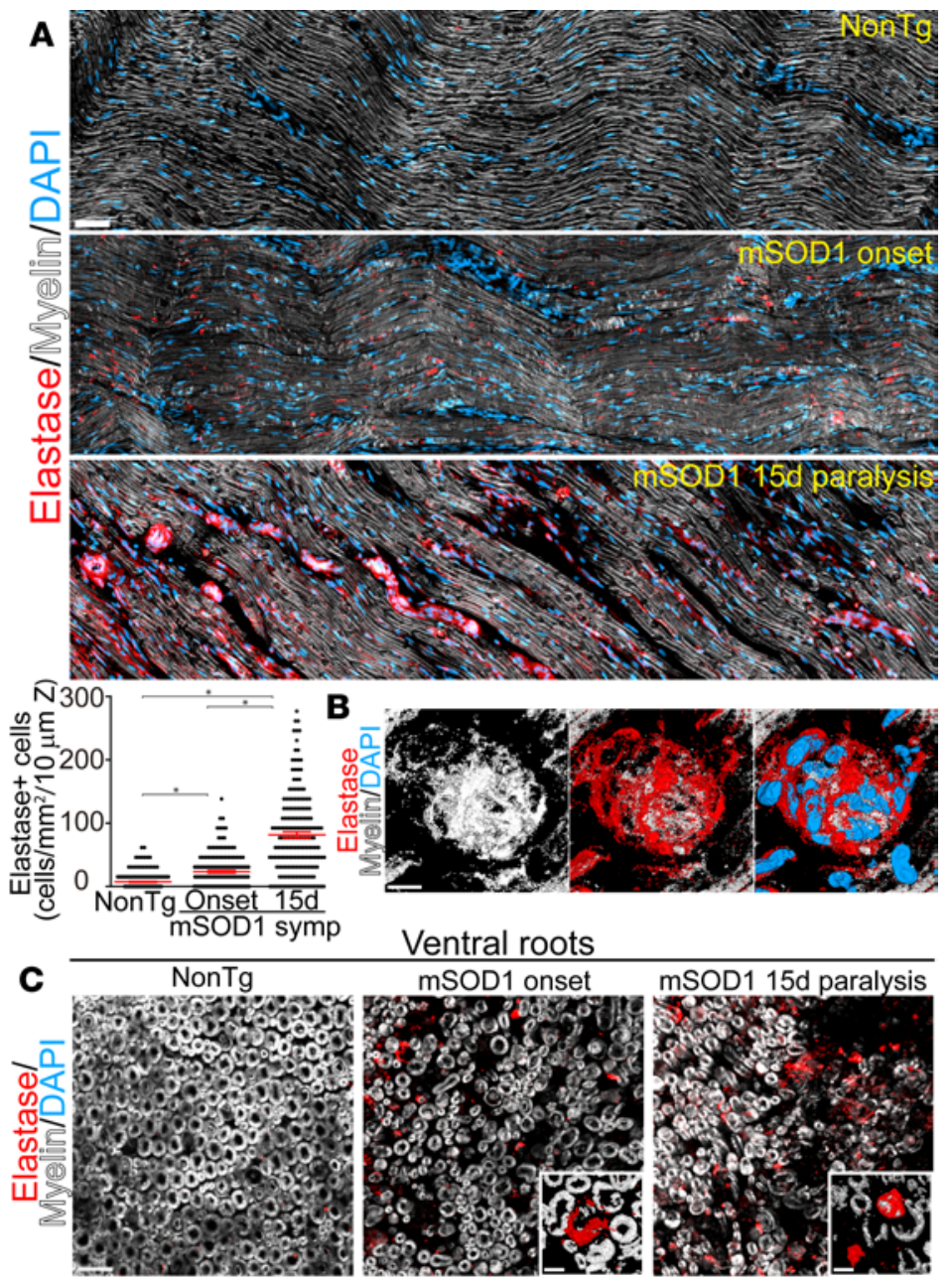

Figure 5. Neutrophils infiltrate into the degenerating sciatic nerve and ventral roots of mSOD1 symptomatic rats. (A) Representative confocal tile reconstruction of $10-\mu \mathrm{m}$ sciatic nerve sections comparing elastase-positive neutrophil (red) infiltration among conditions. Note the significant number of neutrophils that infiltrate during disease progression when compared with nontransgenic (NonTg) rats, where no neutrophils are observed. The number of elastase-positive cells increases as disease progresses. Scale bar: $50 \mu \mathrm{m}$. Note the significant increase of neutrophils after onset, correlating with paralysis progression represented in the graph below. Data are expressed as the mean \pm SEM and were analyzed by Kruskal-Wallis followed by Dunn's multiple-comparisons test ( $n=4$ animals/condition); ${ }^{*} P<0.05$. (B) During advanced paralysis, neutrophils aggregate and are observed phagocytizing myelin ovoids (white). Scale bar: $10 \mu \mathrm{m}$. (c) Ventral roots were analyzed for neutrophil infiltration among conditions. No elastase-positive neutrophils where observed in NonTg rats; however, substantial infiltration of elastase-positive neutrophils (red, arrows) is observed at disease onset and increases during paralysis progression. Myelin ovoid (white) phagocytosis by neutrophils (red) is commonly observed in ventral roots during disease progression (insets). Scale bars: $25 \mu \mathrm{m}$ in low-magnification panels and $10 \mu \mathrm{m}$ in insets.

mast cells to peripheral motor pathway pathology, we administered oral masitinib (30 mg/kg/d) to SOD $1^{\mathrm{G} 93 \mathrm{~A}}$ rats for 15 days after paralysis onset and then measured muscle and nerve pathology by analyzing inflammatory cell recruitment. Postparalysis masitinib treatment significantly reduced the infiltration of elastase-positive neutrophils surrounding the motor nerve endings of the EDL muscle analyzed in whole mounted preparations (Figure 6, A and C). Figure 6B shows representative transversal sections of the EDL muscle stained with anti-myosin heavy chain to identify type $2 \mathrm{~B}$ fast myofibers, muscle cells that exhibit faster contraction kinetics and greater force generation, the number of which drastically decrease during ALS (38). Masitinib treatment significantly prevented the loss of 2B fibers (Figure 6B). Also, masitinib treatment decreased mast cell infiltration into the sciatic nerve as compared with vehicle-treated controls by $40 \%$ (Figure 6D) and also decreased degranulation of mast cells by $60 \%$ (Figure $6 \mathrm{E}$ ). Additionally, masitinib treatment downregulated neutrophil infiltration into the sciatic nerve by $50 \%$ (Figure $6 \mathrm{~F}$ ), further suggesting the concerted activation of both mast cells and neutrophils in mediating distal axon pathology.

Masitinib-induced reduction of immune cell infiltration was associated with preserved nerve pathology. We tested whether downregulation of immune cell infiltration by masitinib could modulate the Wallerian-like pathology at the sciatic nerve during paralysis progression. Figure 7A and Supplemental Figure 4 show that, at 15 days after paralysis onset, a significant number of axons were lost and replaced by myelin ovoids along the peripheral motor pathway. In ventral roots, axon numbers decreased by $45 \%$ between paralysis onset and advanced paralysis (76.4 \pm 3 and $30.1 \pm 2$, respectively, compared with NonTg rats) (Figure 7A). Notably, masitinib treatment significantly prevented postparalysis axonal degeneration in ventral roots and sciatic nerve compared with vehicle-treated rats by $30 \%$ and $20 \%$, respectively (Figure 7A and Supplemental Figure 4). The protection of sciatic nerve pathology by masitinib was also evidenced by the estimate of demyelinating Schwann cells, which typically express 
A

EDL muscle - mSOD1 15d paralysis
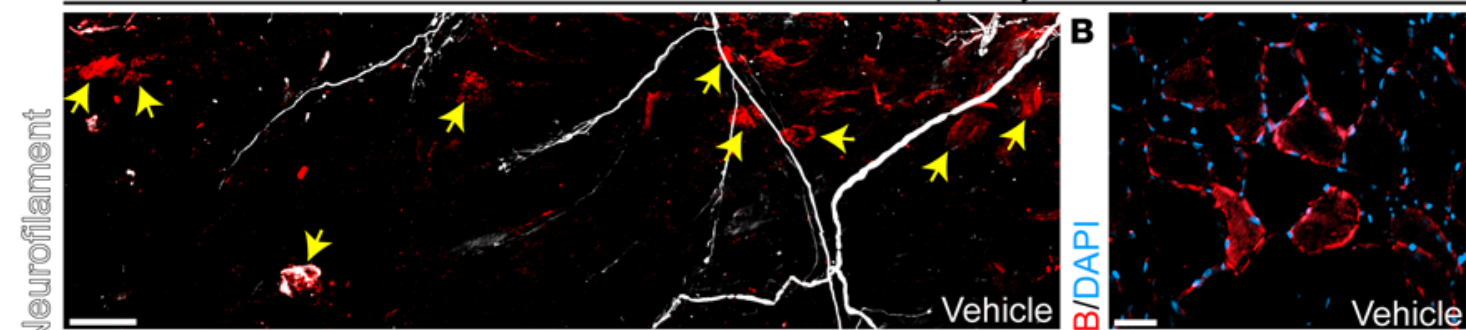

ब্
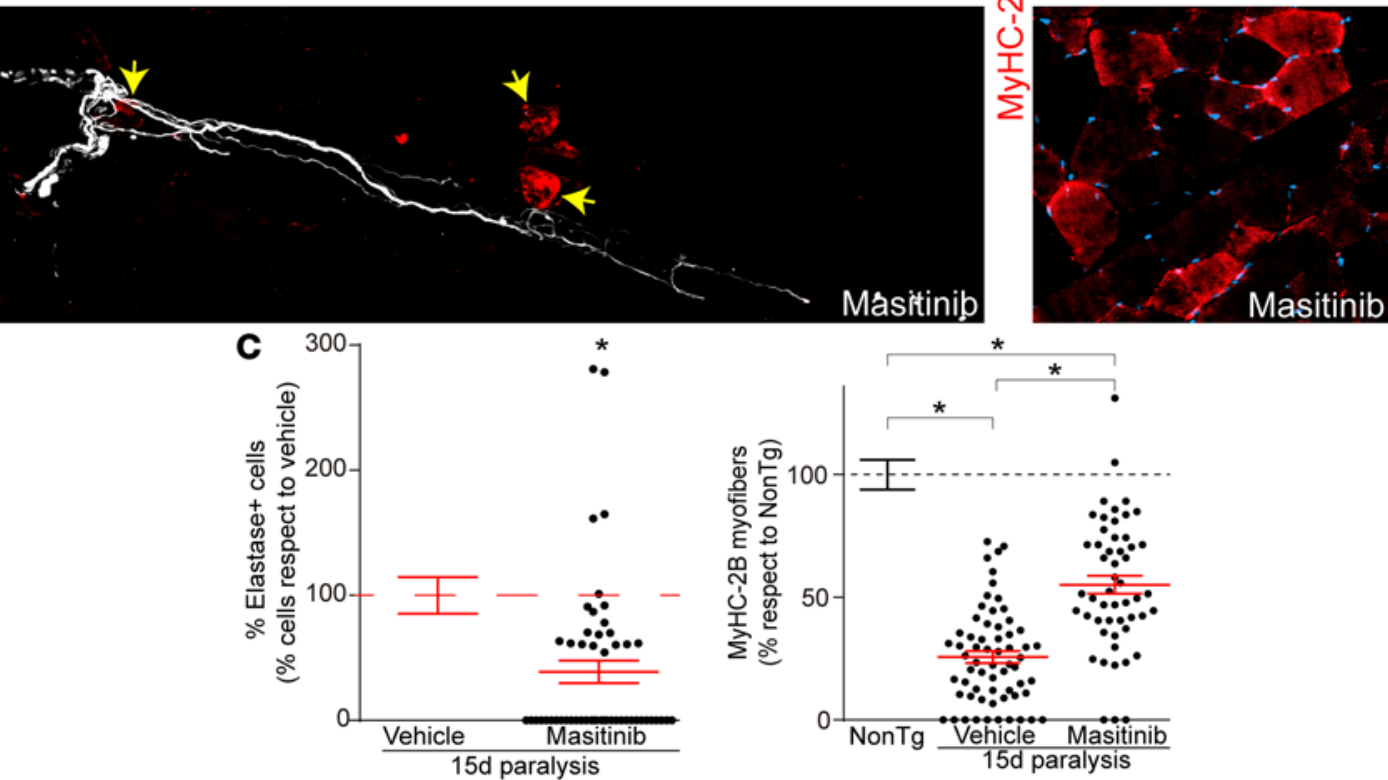

Sciatic nerve - mSOD1 15d paralysis
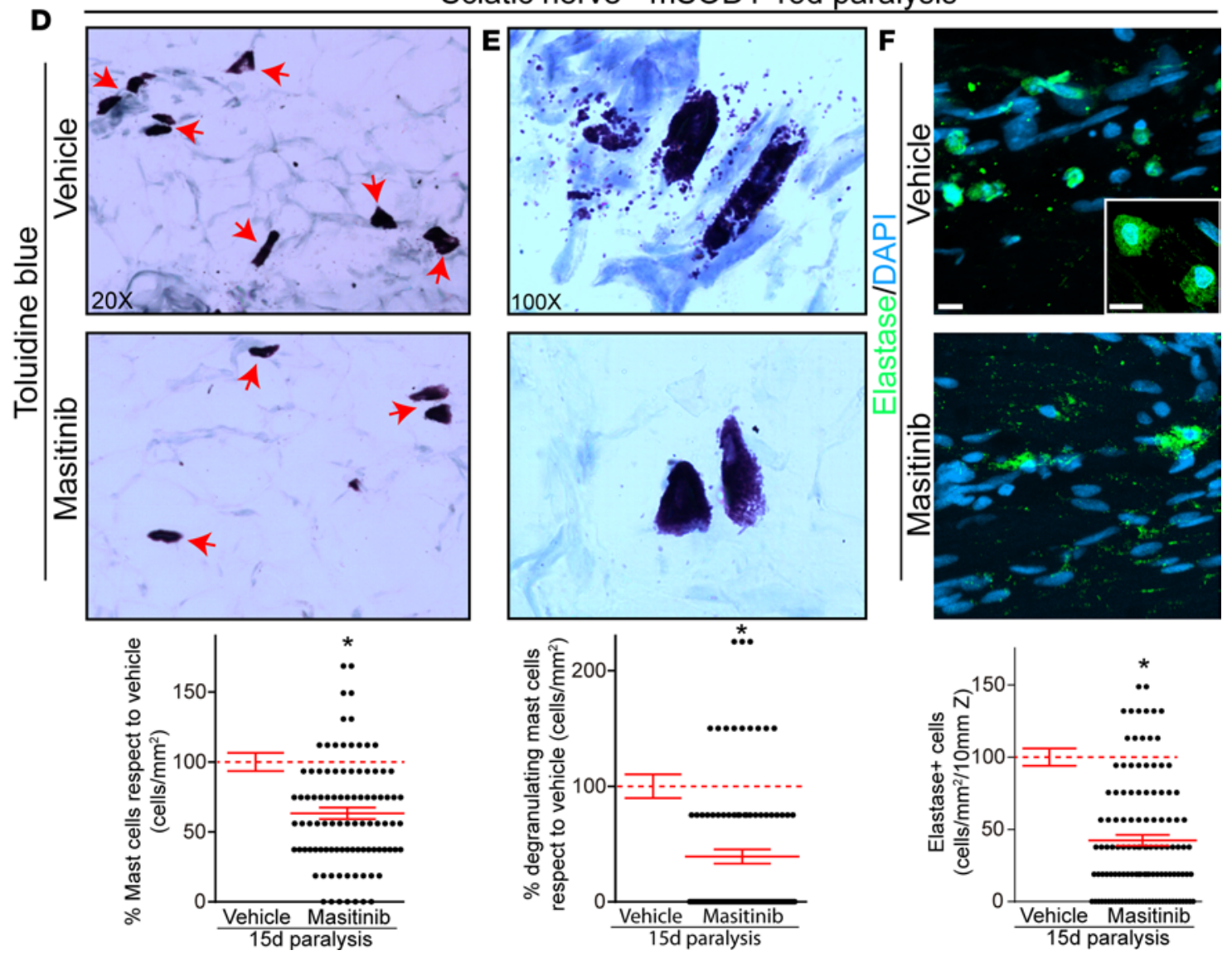
Figure 6. Masitinib treatment reduces mast cell and neutrophil infiltration into the sciatic nerve and prevents muscle atrophy in mSOD1 rats. Masitinib (30 mg/kg/day) or vehicle was orally administered for 15 days after paralysis onset and rat EDL muscle and sciatic nerves were processed for histochemical analysis. (A) Representative confocal images showing the infiltration of elastase-positive neutrophils (red, yellow arrows) in the surroundings of motor nerve terminals. Scale bar: $100 \mu \mathrm{m}$. (B) Transverse cryosections of EDL rat muscle showing staining with an anti-myosin heavy chain 2B isoform (MyHC-2B, red). Masitinib treatment reduced the loss of 2B myofibers. Scale bar: $20 \mu \mathrm{m} . n=3$ animals/condition (20 muscle slices per animal analyzed). (C) Left graph shows the quantitative analysis of elastase-positive neutrophils infiltrating masitinib-treated rats compared with vehicle-treated ones. $n=4$ animals/condition. Right graph shows the quantification of 2B myofibers in EDL muscle among conditions. The number of MyHC-2B-positive fibers from nontransgenic (NonTg) animals was considered $100 \% . n=3$ animals/condition. (D and E) Representative images of sciatic nerve stained with toluidine blue, showing the number and degranulation of mast cells. The graphs below show the quantitative analysis of the number of total mast cells (D) and degranulating mast cells (E) assessed in toluidine blue-stained sections. $n=4$ animals/condition. (F) Representative confocal microphotographs showing elastase-positive neutrophils infiltrating symptomatic vehicle- and masitinib-treated sciatic nerves. Graph below shows the quantitative analysis of the total number of elastase-positive neutrophils infiltrating the sciatic nerve compared with vehicle-treated rats. Scale bars: 20 $\mu \mathrm{m}$ (low-magnification panels) and $10 \mu \mathrm{m}$ (high-magnification inset). $n=4$ animals/condition. Data are expressed as the mean \pm SEM and were analyzed by 2-tailed Mann-Whitney test (C, left; and $\mathbf{D}-\mathbf{F})$ or Kruskal-Wallis followed by Dunn's multiple-comparisons test (C, right). ${ }^{*} P<0.05$.

GFAP and S100B and peak after paralysis onset (Figure 7B). Masitinib treatment decreased GFAP and S100B by approximately $60 \%$ and $70 \%$, respectively, as compared with vehicle-treated rats.

\section{Discussion}

Here, we report a significant and correlated infiltration of degranulating mast cells and neutrophils into skeletal muscle from ALS patients, the significance of which has not been previously recognized. These cells interact with each other, myofibers, and motor endplates, suggesting a concerted inflammatory reaction associated with NMJ denervation and muscle atrophy in ALS. Mast cells, phagocytic neutrophils, and NETs are also abundant around NMJs and along degenerating axons in ventral roots and sciatic nerves, the infiltration being strikingly associated with paralysis progression. These findings further implicate these immune effector cells in driving ALS progression. In accordance, pharmacological downregulation of mast cell infiltration by the tyrosine kinase inhibitor masitinib in SOD1 ${ }^{\mathrm{G} 93 \mathrm{~A}}$ rats significantly reduces immune cell infiltration, resulting in decreased muscle and axonal pathology and myelin disruption. In agreement with previous reports $(10,12,31)$, the present findings provide insight into immunopathogenic mechanisms mediating peripheral motor pathway degeneration in ALS and underscores potential therapeutic approaches to delay disease progression.

While mast cells infiltrate the EDL muscle in SOD $1{ }^{\mathrm{G} 93 \mathrm{~A}}$ rats after paralysis onset (10), we found a comparable increased mast cell number in the quadriceps muscles from subjects with ALS. Three out of 5 patients analyzed displayed a simultaneous pronounced increase in both mast cell and neutrophil numbers with respect to controls, while 2 others showed a moderate increase. The variability in mast cell and neutrophil infiltration among the ALS muscle samples may be related to the clinical or pathological heterogeneity of the disease. While all had premortem evidence of denervation in the autopsied muscle (as determined by EMG), patients had variable clinical deficits and regions of ALS onset. The 2 muscle samples with lower mast cell infiltration were from patients in whom the disease started in the arm and bulbar region. Moreover, one of those patients had severe spasticity and rigidity in the quadriceps muscle, reflecting predominant upper motor neuron dysfunction. Additional testing from patients spanning the clinical and pathological spectrum of ALS should provide further insight into the role of mast cells and neutrophils in disease progression.

The correlation between mast cell and neutrophil numbers further suggests that these cells act in concert, with mast cells being recruited in response to motor nerve terminal degeneration and/or myofiber denervation. In turn, mast cells have the potential to orchestrate neutrophil recruitment $(39,40)$. Previous reports have shown mast cell infiltration in patients with muscular dystrophy and autoimmune myositis $(17,41-43)$. Although our study has the limitation of analyzing autopsied tissues from a limited number of ALS subjects having different clinical features, comparison with non-ALS controls reveals remarkable differences in the pattern of immune cell infiltration. First, the density and size of mast cells were significantly increased in ALS patients with respect to controls. Second, mast cell degranulation was frequently found in ALS patients but not in controls. Finally, mast cells in ALS were in close proximity to myofibers as compared with the perivascular location in controls. Mast cell degranulation in muscles from ALS patients suggests multiple local responses mediated by secreted proteases, cytokines, trophic factors, and vasoactive mediators (44). While mast cells may promote muscle repair in healthy individuals, they can directly or indirectly trigger damage of myofibers, motor nerve endings, Schwann cells, and capillary networks, as well as promote fibrosis and collagen deposition $(45,46)$. An active fibrotic process has been reported in skeletal 


\section{A}
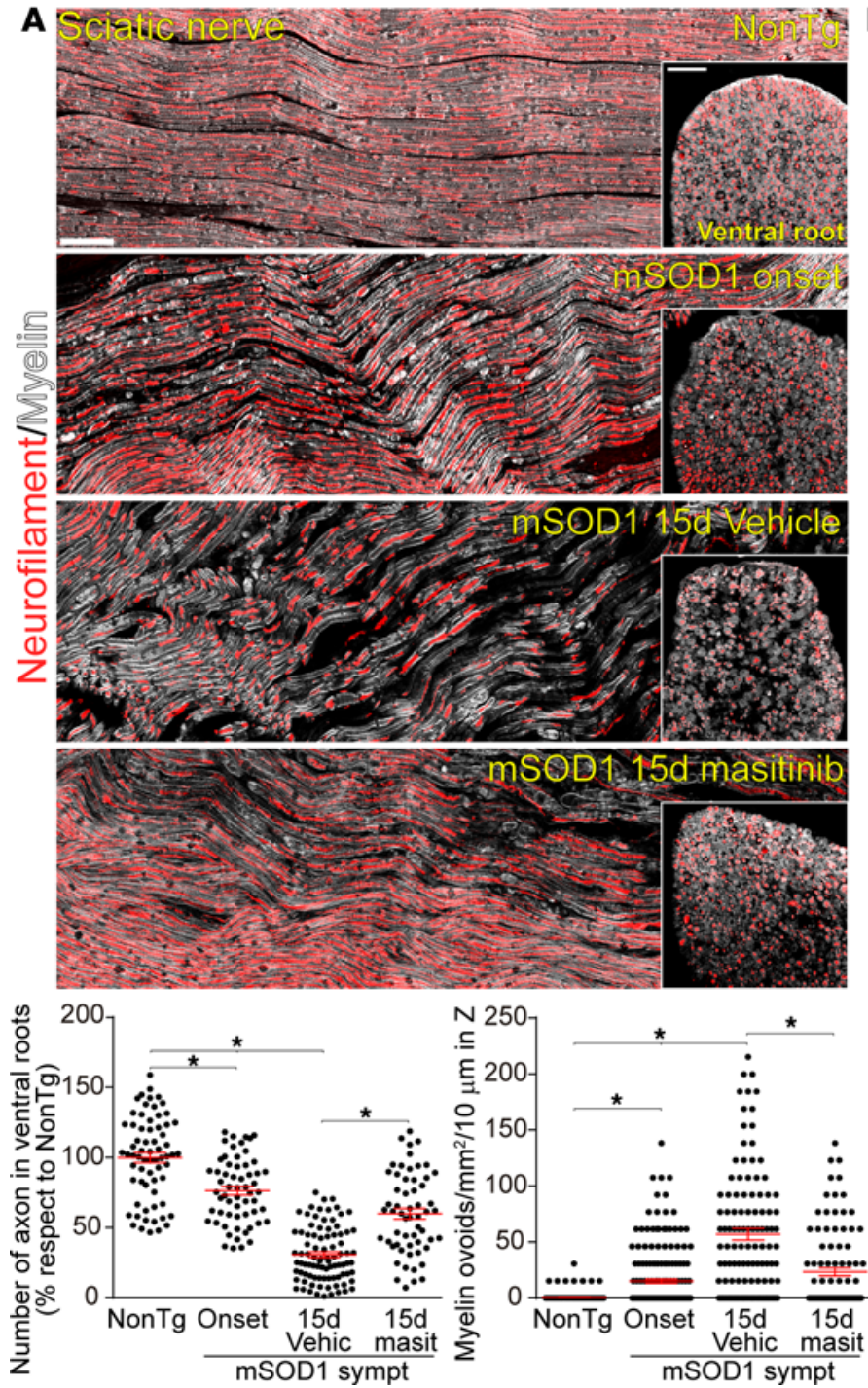
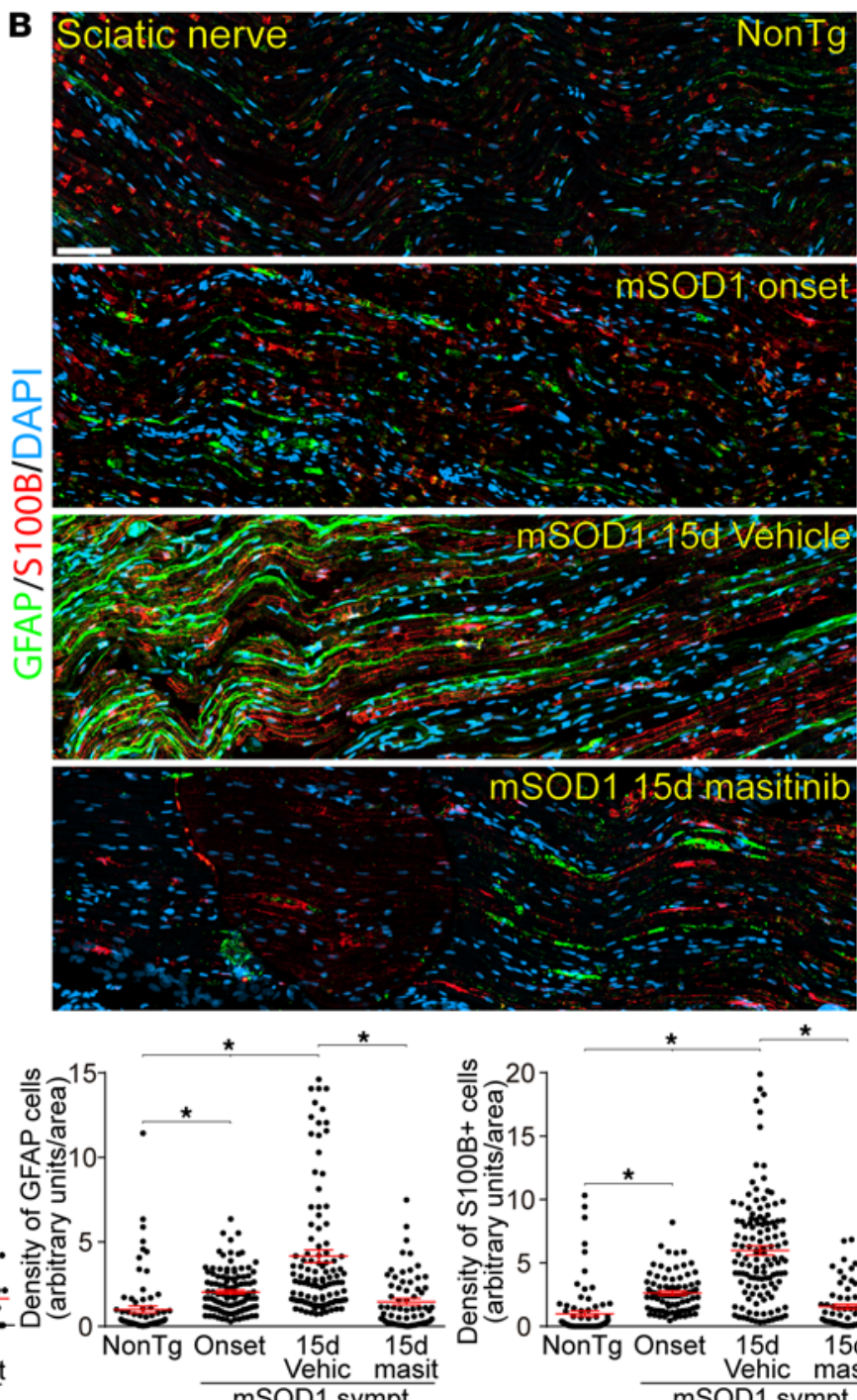

mSOD1 sympt

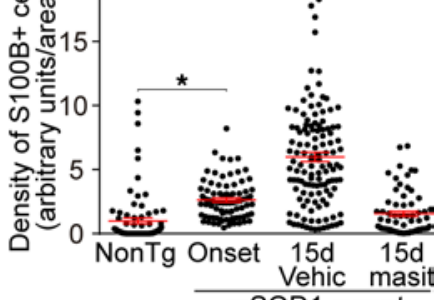

mSOD1 sympt

Figure 7. Postparalysis treatment with masitinib prevents axonal and myelin degeneration and Schwann cell reactivity in the degenerating sciatic nerve and ventral root of SOD1 ${ }^{\mathrm{C93A}}$ rats. Confocal representative images of sciatic nerves and ventral roots of nontransgenic (NonTg) and SOD1 ${ }^{\mathrm{C9} 3 \mathrm{~A}}$ (onset, 15 -day paralysis-vehicle and 15-day paralysis-mastitinib). Masitinib (30 mg/kg) was administered after paralysis onset and continued for 15 days. (A) Sciatic nerve sections $(10 \mu \mathrm{m}$ ) were stained for neurofilaments (red) or myelin (white) to analyze axonal and myelin structure among conditions. Scale bar: $50 \mu \mathrm{m}$. Insets show a representative confocal image of ventral root cross sections for each condition stained for neurofilament (red) and myelin (white). Number of axons per root area as well as myelin ovoids in the sciatic nerve were analyzed and plotted in the left and right graphs, respectively. $n=4$ animals/condition for sciatic nerve analysis and $n=3$ animals/condition for ventral root analysis. (B) Representative sciatic nerve confocal images showing staining for Schwann cells, GFAP (green), and S100B (red). Few Schwann cells are observed in NonTg animals and S100B signal is restricted to Schmidt-Lanterman clefts. A significant glial reactivity is observed as disease progresses. Masitinib treatment significantly prevents Schwann cell reactivity. Scale bar: $50 \mu \mathrm{m}$. $n=4$ animals/ condition. Graphs below show the quantitative analysis of the density of Schwann cells per area. All quantitative data are expressed as the mean \pm SEM and were analyzed by Kruskal-Wallis followed by Dunn's multiple-comparisons test. ${ }^{*} P<0.05$.

muscle of symptomatic SOD1 ${ }^{\mathrm{G} 93 \mathrm{~A}}$ mice (47). Thus, abnormally activated mast cells in ALS muscles likely contribute to myofiber damage, NMJ degradation, and fibrotic muscle remodeling.

The accumulation of endomysial elastase-expressing neutrophils in muscles from both ALS subjects and SOD $1^{\mathrm{G} 93 \mathrm{~A}}$ rats is intriguing. Neutrophil elastase activity is upregulated in muscular dystrophy and reported to impair myogenesis (48), suggesting a role for neutrophil-mediated inflammation in muscle atrophy. Because NET formation denotes neutrophil hyperactivation with a high cytotoxic potential (30), NETs in ALS subjects suggest the cells behave as cytotoxic effectors mediating damage and degradation of neuromuscular components. In accordance, we found that neutrophils progressively accumulate close to motor nerve axon bundles and motor endplates and actively participate in phagocytizing degenerating axons. Previous reports indicate neutrophil-mediated myofiber damage and muscular atrophy in several pathological 
conditions including inflammatory myopathies (49) and muscle functional remodeling $(27,50,51)$. Neutrophil depletion in Duchenne muscular dystrophy reduces myofiber loss (52), further indicating their role as cytotoxic effectors. Previous studies have reported an increase in the blood neutrophil/monocyte ratio and in the absolute number of circulating neutrophils in ALS patients, strongly correlating with rapid disease progression $(53,54)$. Neutrophils have also been implicated as cytotoxic effectors mediating neuronal damage in Alzheimer's disease $(23,24)$. In this context, our results strongly indicate that the invasion or uncontrolled activation of neutrophils contributes to distal motor axon and muscular pathology ALS.

Mast cells and neutrophils also infiltrate into the endoneurium of the sciatic nerve up to the ventral roots, with a time course similar to that observed for mast cells and macrophages in skeletal muscle of SOD1 ${ }^{\mathrm{G} 93 \mathrm{~A}}$ rats (10). This pathological process has not been previously recognized in ALS. Chymase released by mast cells is a well-recognized chemoattractant for neutrophils $(20,55)$, and thus it may underlie the remarkable association between both cells. In addition, mast cells can release a variety of vasoactive and inflammatory factors that can orchestrate deleterious effects in ALS (56-58). Our findings raise an important causality dilemma concerning the pathogenic significance of immune cell recruitment in the neuromuscular compartment in ALS. Is it a primary cause of peripheral nerve degeneration or rather a secondary adaptive response to neurodegeneration? While this question is not completely answered in our study, a number of reports suggest that chronic and exacerbated inflammation in the peripheral motor pathway would be deleterious in $\operatorname{ALS}(11,12,59,60)$. Moreover, chronic nerve damage mediated by invading immune cells may also prevent motor axon regeneration or myelination or even mediate damage of sensory axons underlying small fiber neuropathy previously recognized in ALS patients (61).

In accordance with previous reports showing that targeting immune cells with tyrosine kinase inhibitor drugs prevents NMJ denervation and delays postparalysis survival $(10,12,31)$, we found that masitinib downregulates mast cell and neutrophil infiltration along the peripheral motor pathway and prevents spinal nerve pathology. c-Kit and CSF-1R receptors are key molecular targets of masitinib $(31,33)$. Accordingly, we found c-Kit-expressing mast cells in autopsied muscles from ALS patients and SOD1 ${ }^{\mathrm{G} 93 \mathrm{~A}}$ rats, further indicating an antiinflammatory mechanism of action. The molecular pathways underlying the drug effect on neutrophil infiltration have not been determined; however, the effect could be secondary to a masitinib-mediated reduction in mast cells and macrophages through c-Kit and CSF-1R inhibition. Moreover, postparalysis treatment with masitinib significantly decreased axonal pathology, myelin disruption, and Schwann cell activation, further supporting its neuroprotective effect. In addition, masitinib treatment potently prevented the loss of type 2B fast myofibers in the EDL muscle, an effect likely indicating a decrease in NMJ denervation. Although our study does not unambiguously determine whether the protective effects of masitinib are primarily exerted in the PNS or CNS, it provides further evidence for a multifaceted effect of the drug in downregulating immune cells along the motor pathways.

In conclusion, the present study shows that mast cells and neutrophils are abundant along the peripheral motor pathway in ALS. These cells appear to be relevant immune cytotoxic effectors in ALS with the potential to be pharmacologically targeted by tyrosine kinase inhibitor drugs. Finally, we show further evidence for masitinib ameliorating the peripheral motor axon pathology in SOD1 ${ }^{\mathrm{G} 93 \mathrm{~A}}$ rats, providing a mode of action to explain the promising therapeutic effect of the drug in ALS.

\section{Methods}

Animals. Male SOD1 ${ }^{\mathrm{G} 93 \mathrm{~A}}$ progeny, purchased from Taconic Bioscience (NTac:SD-Tg(SOD1G93A)L26H), were used for further breeding to maintain the line. Rats were housed in a centralized animal facility with a 12-hour light-dark cycle with ad libitum access to food and water. Perfusion with fixative was performed under $90 \%$ ketamine and 10\% xylazine anesthesia and all efforts were made to minimize animal suffering, discomfort, or stress.

Experimental conditions. At least 4 male rats were analyzed for each experiment. Four different conditions were studied as follows: (a) NonTg rats at 160-180 days of age, (b) transgenic SOD $1^{\mathrm{G} 93 \mathrm{~A}}$ rats at 180-190 days of age (symptomatic, onset), (c) transgenic SOD ${ }^{\mathrm{G} 93 \mathrm{~A}}$ rats at 195-210 days of age treated with vehicle (15-day paralysis-vehicle), and (d) transgenic SOD1 ${ }^{\mathrm{G} 93 \mathrm{~A}}$ rats at 195-210 days of age treated with $30 \mathrm{mg} / \mathrm{kg} / \mathrm{d}$ of masitinib for 15 days (15-day paralysis-masitinib).

Determination of disease onset and end stage. As described previously (31), all rats were weighed and evaluated for motor activity daily. Disease onset was determined for each animal when pronounced 
muscle atrophy was accompanied by abnormal gait, typically expressed as subtle limping or dragging of one hind limb. When necessary, end stage was defined by a lack of righting reflexes or the inability to reach food and water.

Masitinib administration. As described previously (31), only transgenic rats showing weakness and gait alterations in hind limbs as first clinical sign were selected for masitinib treatment studies. Rats were divided randomly into the masitinib- or vehicle-treated groups. Masitinib mesylate (AB Science, AB1010), freshly prepared in sterilized drinking water, was administered daily at a dose of $30 \mathrm{mg} / \mathrm{kg}$ using a curved stainless steel gavage needle with 3-mm ball tip. Dosing was defined in accordance with previous studies in the same rat model of ALS that was shown to be safe for chronic treatments (31). Rats were treated from day 1 after paralysis for an additional 15 days, after which they were euthanized.

Human tissue collection. The collection of postmortem human ALS and control samples was approved by the University of Alabama, Birmingham (UAB) Institutional Review Board. All ALS patients were cared for at UAB and so detailed clinical records were available. Control samples were age matched and were harvested from patients who expired from non-neurological causes. The average collection time after death was less than 10 hours. All tissues were harvested by PHK and YS at the time of autopsy and preserved within 30 minutes

Human muscle immunohistochemistry. Quadriceps paraffin sections $(10 \mu \mathrm{m})$ were sliced using a microtome. Following deparaffinization, slices were blocked and permeabilized in 5\% BSA/0.5\% Triton X-100 for 2 hours at room temperature. Primary antibodies were incubated in 1\% BSA/0.5\% Triton X-100 at $4^{\circ} \mathrm{C}$ overnight. After washing, secondary antibodies were incubated for 3 hours at room temperature. After PBS washing, Mowiol medium (Sigma-Aldrich) was used for mounting. Elastase-positive single cells were counted in at least 20 fields per section (at least three 10- $\mu \mathrm{m}$ sections of each quadriceps muscle) per patient or control. Quantitative analysis was performed using ImageJ software (NIH) and the total number of neutrophils per area was plotted.

Immunohistochemistry of rat whole mounted muscle. As previously described (10), rats were deeply anesthetized using $90 \%$ ketamine and 10\% xylazine anesthesia and fixed by perfusion with $4 \%$ paraformaldehyde in PBS. EDL muscles from the hind limb were dissected. Then, tissues were blocked for 2 hours at room temperature in $5 \% \mathrm{BSA} / 1 \%$ Triton X-100 in PBS, and incubated with primary antibodies at $4^{\circ} \mathrm{C}$ overnight. After washing, secondary antibodies were incubated overnight at $4^{\circ} \mathrm{C}$, washed with PBS 3 times for 15 minutes each, and whole mounted in Mowiol mounting medium on slides. Elastase-positive neutrophils were counted in whole mounted EDL muscle using a stereological approach, contrasting neutrophil number in the region surrounding the motor endplates (NMJ compartment). At least 30 confocal stacks $(320 \times 320 \times 100 \mu \mathrm{m}$ in $Z)$ from 3 animals among conditions were analyzed as shown in Figures 3 and 6.

Immunohistochemistry of fast $2 B$ isotype myofibers in EDL rat muscle. EDL muscles from 3 different animals per condition were embedded in TissueTek (Sakura) and sectioned using a cryostat, collecting $10-\mu \mathrm{m}$ slices in gelatin-coated slides. Muscle sections were blocked for 2 hours at room temperature in 5\% BSA/0.5\% Triton X-100 in PBS, and incubated with primary antibodies for 3 hours at room temperature in $1 \%$ BSA $/ 0.3 \%$ Triton X-100. After washing, secondary antibodies were incubated for 2 hours at room temperature in 1\% BSA $/ 0.3 \%$ Triton X-100. Mowiol was used for mounting. Analysis was carried out using a Zeiss LSM 800 confocal with a $\times 20$ objective. At least 20 fields per muscle from 3 rats per group were acquired for counting. The number of 2B-positive fibers from NonTg rats was considered 100\%. Quantitative analysis was performed using the ImageJ program and the ratio of fast $2 \mathrm{~B}$ myofibers relative to total myofibers in NonTg was plotted.

Immunohistochemistry of sciatic nerve and ventral roots. Paraformaldehyde-fixed (4\%) sciatic nerve and ventral roots were cryopreserved in $30 \%$ sucrose (Sigma-Aldrich) at $4^{\circ} \mathrm{C}$. After 72 hours, tissue was embedded in TissueTek, sectioned (longitudinal) using a cryostat, and collected on gelatin-coated slides. Sections $(10 \mu \mathrm{m})$ were blocked for 2 hours at room temperature in 5\% BSA/0.5\% Triton X-100 in PBS and then incubated with primary antibodies overnight at $4^{\circ} \mathrm{C}$ in $1 \% \mathrm{BSA} / 0.3 \%$ Triton $\mathrm{X}-100$. After washing, secondary antibodies were incubated for 2 hours at room temperature in $1 \%$ BSA/0.5\% Triton X-100. Mowiol was used for mounting. Using a stereological approach, tryptase-positive mast cells and elastase-positive neutrophils were counted in $10-\mu \mathrm{m}$ sciatic nerve sections using confocal microphotographs with a magnification of $\times 25$. At least 20 sections per sciatic nerve per animal were analyzed $(n=4)$. ImageJ software was used for analysis. For axonal structure and Schwann cell density analysis, neurofilament and GFAP/S100B density analysis was measured using ImageJ. At least 20 
sections per sciatic nerve per animal $(n=4)$ were analyzed as shown in Figure 7. Myelin ovoids were counted in at least 20 sections per nerve per animal among conditions $(n=4)$, as previously described (62). Axon number in cross sections of ventral roots was determined by assessment on confocal $\times 25$ microphotographs. Neurofilament-positive axons were counted as intact if neurofilament labeling was confined to and filled the bulk of the axonal structure. Myelin staining was used to further analyze intact axon structure. At least 20 slides from 3 different rats were analyzed and the number of axons per area was plotted.

Antibodies used. Mouse monoclonal anti-chymase (Abcam, ab2377, 1:250), 1:200 goat polyclonal anti-chymase (Abcam, ab111239), 1:250 rat polyclonal anti-c-Kit (biotin) (Abcam, ab25022), 1:250 mouse monoclonal anti-c-Kit (Abcam, ab187371), 1:200 mouse monoclonal anti-tryptase (Abcam, ab2378), 1:350 rabbit polyclonal anti-neutrophil elastase (Abcam, ab21595), 1:150 rabbit polyclonal anti-MPO (Abcam, ab9535), 1:400 rabbit polyclonal anti-GFAP (Sigma-Aldrich, G9269), 1:300 mouse monoclonal anti-S100B (Sigma-Aldrich, S2532), 1:300 mouse monoclonal anti-neurofilament 200Alexa Fluor 555 (Thermo Fisher Scientific, MAB5256A5), 1:500 $\alpha$-bungarotoxin-Alexa Fluor 488 (Thermo Fisher Scientific, B13422), 1:300 myelin-Alexa Fluor 488 (Thermo Fisher Scientific, F34651), and 1:40 mouse IgM anti-myosin heavy chain type 2B (DSHB, BF-F3). Secondary antibodies: 1:500 goat anti-rabbit-Alexa Fluor 488 or -Alexa Fluor 546 (Thermo Fisher Scientific, A11035 or A11034), 1:500 goat anti-mouse-Alexa Fluor 488, Alexa Fluor 546, or Alexa Fluor 633 (Thermo Fisher Scientific, A11029, A11030, or A21052), 1:500 goat anti-mouse IgM-Alexa Fluor 488 (Thermo Fisher Scientific, A21042), 1:500 Streptavidin-Alexa Fluor 405 or Alexa Fluor 633 (Thermo Fisher Scientific, S32351 or S21375).

Toluidine blue staining of human muscle slices and rat sciatic nerves, dorsal root ganglia, and spinal cord. For the mast cell analysis based on metachromasia observation, as previously described (10), 10- $\mu \mathrm{m}$ sections of quadriceps human muscle (paraffin sections) and rat sciatic nerves, dorsal root ganglia and spinal cord (fixed frozen sections) were microtome or cryostat sliced and mounted in gelatin-coated slides, respectively. Human muscle slices were deparaffinized before starting staining. Sections were washed and hydrated 2 times in distilled water for 10 minutes and embedded in $1 \%$ toluidine blue solution for 10-20 minutes. Then, slides were washed in distilled water 3 times 5 minutes and dehydrated for 3 minutes in 70\% ethanol, 3 minutes in 95\% ethanol, and finally 2 minutes in $100 \%$ ethanol. Slides were cleared in xylene twice, 3 minutes each, and finally mounted in DPX (Sigma-Aldrich). Images (at $\times 10, \times 20$, and $\times 100$ magnification) were acquired using an Olympus CX41 microscope connected to a EvolutionLC Color camera and using ImagePro Express software for acquisition. For human mast cell counting, at least 35 fields per quadriceps muscle (5 control and 5 ALS patients) were analyzed using a magnification of $\times 20$. The number of toluidine blue-positive metachromatic mast cells in the SOD $1{ }^{\mathrm{G} 93 \mathrm{~A}}$ rat model was counted using ImageJ software, in at least 20 fields per sciatic nerve $(1 \mathrm{~cm}$ long) per animal using a magnification of $\times 10$. For dorsal root ganglion and spinal cord analysis, at least ten 8 - $\mu \mathrm{m}$ sections from 3 different rats among groups were stained and analyzed for toluidine blue-positive mast cells. Degranulating rat mast cells, characterized by extensive metachromatic granules being released by an isolated cell, were counted using $\times 20$ magnification in at least 20 serial pictures of each sciatic nerve section $(n=4)$. Human and rat data are expressed as the number of mast cells per $\mathrm{mm}^{2}$.

Fluorescence imaging. Fluorescence imaging was performed with a laser scanning Zeiss LSM 800 confocal microscope with either a $\times 25$ (1.2 numerical aperture) objective or $\times 63$ (1.3 numerical aperture) oil-immersion objective using Zeiss Zen Black software. Maximum intensity projections of optical sections were created with Zeiss Zen software.

Statistics. Quantitative data are expressed as the mean \pm SEM. Two-tailed Mann-Whitney test or Kruskal-Wallis followed by Dunn's multiple-comparisons test were used for statistical analysis, with $P$ less than 0.05 considered significant. GraphPad Prism 7.03 software was used for statistical analyses.

Study approval. All procedures using laboratory animals were performed in accordance with national and international guidelines, and in strict accordance with the Institut Pasteur de Montevideo Ethical Committee for the use of Experimental Animals (CEUA) requirements and under the current ethical regulations of Uruguayan Law $\mathrm{N}^{\circ} 18.611$ for animal experimentation that follows the Guide for the Care and Use of Laboratory Animals of the NIH. All experimental procedures were approved by the CEUA of the Institut Pasteur de Montevideo. 


\section{Author contributions}

ET, PHK, ICM, OH, and LB designed the study. ET, PHK, YS, YK, VV, SI, MK, and ICM performed experiments. ET, PHK, YS, ICM, JSB, and LB analyzed data. ET, PHK, ICM, JSB, OH, and LB prepared the manuscript. All authors contributed to discussion of the results and edited and approved the final version of the manuscript.

\section{Acknowledgments}

This work was supported by Institut Pasteur de Montevideo - FOCEM Mercosur (COF 03/11), the Amyotrophic Lateral Sclerosis Association (00482), Agencia Nacional de Investigación e Innovación (ANII), NINDS R01NS092651 (to PHK), and a Merit Review BX001148 from the Department of Veterans Affairs (to PHK), Programa de Desarrollo de las Ciencias Básicas (PEDECIBA), Sistema Nacional de Investigadores (SNI, Uruguay), ECOS-Sud Program (U014S02), and Thierry Latran in his capacity as Founder of Foundation Thierry Latran. Also, we thank Colin Mansfield for his critical comments and helpful suggestions in revising the manuscript and the staff from the Transgenic and Experimental Animal Unit from Institut Pasteur de Montevideo. We are grateful to our ALS patients who donated their skeletal muscle tissues postmortem to advance ALS research.

Address correspondence to: Luis Barbeito, Institut Pasteur de Montevideo, Mataojo 2020, Montevideo 11.400, Uruguay. Phone: 00.598.2.522.0910; Email: barbeito@pateur.edu.uy.

1. Brown RH, Al-Chalabi A. Amyotrophic lateral sclerosis. NEngl J Med. 2017;377(2):162-172.

2. Kennel PF, Finiels F, Revah F, Mallet J. Neuromuscular function impairment is not caused by motor neurone loss in FALS mice: an electromyographic study. Neuroreport. 1996;7(8):1427-1431.

3. Fischer LR, et al. Amyotrophic lateral sclerosis is a distal axonopathy: evidence in mice and man. Exp Neurol. 2004;185(2):232-240.

4. Frey D, Schneider C, Xu L, Borg J, Spooren W, Caroni P. Early and selective loss of neuromuscular synapse subtypes with low sprouting competence in motoneuron diseases. J Neurosci. 2000;20(7):2534-2542.

5. Kong J, Xu Z. Massive mitochondrial degeneration in motor neurons triggers the onset of amyotrophic lateral sclerosis in mice expressing a mutant SOD1. J Neurosci. 1998;18(9):3241-3250.

6. Brady ST, Morfini GA. Regulation of motor proteins, axonal transport deficits and adult-onset neurodegenerative diseases. Neurobiol Dis. 2017;105:273-282.

7. Millecamps S, Julien JP. Axonal transport deficits and neurodegenerative diseases. Nat Rev Neurosci. 2013;14(3):161-176

8. Arbour D, Tremblay E, Martineau É, Julien JP, Robitaille R. Early and persistent abnormal decoding by glial cells at the neuromuscular junction in an ALS model. J Neurosci. 2015;35(2):688-706.

9. Campanari ML, García-Ayllón MS, Ciura S, Sáez-Valero J, Kabashi E. Neuromuscular junction impairment in amyotrophic lateral sclerosis: reassessing the role of acetylcholinesterase. Front Mol Neurosci. 2016;9:160.

10. Trias E, et al. Evidence for mast cells contributing to neuromuscular pathology in an inherited model of ALS. JCI Insight. 2017;2(20):95934.

11. Chiu IM, et al. Activation of innate and humoral immunity in the peripheral nervous system of ALS transgenic mice. Proc Natl Acad Sci USA. 2009;106(49):20960-20965.

12. Martínez-Muriana A, et al. CSF1R blockade slows the progression of amyotrophic lateral sclerosis by reducing microgliosis and invasion of macrophages into peripheral nerves. Sci Rep. 2016;6:25663.

13. Van Dyke JM, Smit-Oistad IM, Macrander C, Krakora D, Meyer MG, Suzuki M. Macrophage-mediated inflammation and glial response in the skeletal muscle of a rat model of familial amyotrophic lateral sclerosis (ALS). Exp Neurol. 2016;277:275-282.

14. Nardo G, et al. Immune response in peripheral axons delays disease progression in SOD1G93A mice. J Neuroinflammation. 2016;13(1):261.

15. Galli SJ, Tsai M, Wershil BK. The c-kit receptor, stem cell factor, and mast cells. What each is teaching us about the others. Am J Pathol. 1993;142(4):965-974.

16. Wernersson S, Pejler G. Mast cell secretory granules: armed for battle. Nat Rev Immunol. 2014;14(7):478-494.

17. Abonia JP, et al. Mast cell protease 5 mediates ischemia-reperfusion injury of mouse skeletal muscle. J Immunol. 2005;174(11):7285-7291.

18. Schmelz M, Zeck S, Raithel M, Rukwied R. Mast cell tryptase in dermal neurogenic inflammation. Clin Exp Allergy. 1999;29(5):695-702.

19. Ashina K, et al. Histamine induces vascular hyperpermeability by increasing blood flow and endothelial barrier disruption in vivo. PLoS One. 2015;10(7):e0132367.

20. He S, Walls AF. Human mast cell chymase induces the accumulation of neutrophils, eosinophils and other inflammatory cells in vivo. Br J Pharmacol. 1998;125(7):1491-1500.

21. Christy AL, Walker ME, Hessner MJ, Brown MA. Mast cell activation and neutrophil recruitment promotes early and robust inflammation in the meninges in EAE. J Autoimmun. 2013;42:50-61.

22. Kolaczkowska E, Kubes P. Neutrophil recruitment and function in health and inflammation. Nat Rev Immunol. 2013;13(3):159-175.

23. Zenaro E, et al. Neutrophils promote Alzheimer's disease-like pathology and cognitive decline via LFA-1 integrin. Nat Med. $2015 ; 21(8): 880-886$ 
24. Baik SH, et al. Migration of neutrophils targeting amyloid plaques in Alzheimer's disease mouse model. Neurobiol Aging. 2014;35(6):1286-1292

25. Dong Y, et al. Neutrophil hyperactivation correlates with Alzheimer's disease progression. Ann Neurol. 2018;83(2):387-405.

26. Pizza FX, Peterson JM, Baas JH, Koh TJ. Neutrophils contribute to muscle injury and impair its resolution after lengthening contractions in mice. J Physiol (Lond). 2005;562(Pt 3):899-913.

27. Toumi H, F'guyer S, Best TM. The role of neutrophils in injury and repair following muscle stretch. J Anat. 2006;208(4):459-470.

28. Sheshachalam A, Srivastava N, Mitchell T, Lacy P, Eitzen G. Granule protein processing and regulated secretion in neutrophils Front Immunol. 2014;5:448.

29. Brinkmann V, et al. Neutrophil extracellular traps kill bacteria. Science. 2004;303(5663):1532-1535.

30. Papayannopoulos V. Neutrophil extracellular traps in immunity and disease. Nat Rev Immunol. 2018;18(2):134-147.

31. Trias E, et al. Post-paralysis tyrosine kinase inhibition with masitinib abrogates neuroinflammation and slows disease progression in inherited amyotrophic lateral sclerosis. J Neuroinflammation. 2016;13(1):177.

32. Dubreuil P, et al. Masitinib (AB1010), a potent and selective tyrosine kinase inhibitor targeting KIT. PLoS ONE 2009;4(9):e7258.

33. Anastassiadis T, Deacon SW, Devarajan K, Ma H, Peterson JR. Comprehensive assay of kinase catalytic activity reveals features of kinase inhibitor selectivity. Nat Biotechnol. 2011;29(11):1039-1045

34. Kiernan MC. Motor neuron disease in 2017: Progress towards therapy in motor neuron disease. Nat Rev Neurol. 2018;14(2):65-66.

35. Jensen L, Jørgensen LH, Bech RD, Frandsen U, Schrøder HD. Skeletal muscle remodelling as a function of disease progression in amyotrophic lateral sclerosis. Biomed Res Int. 2016;2016:5930621.

36. Halova I, Draberova L, Draber P. Mast cell chemotaxis - chemoattractants and signaling pathways. Front Immunol. 2012;3:119.

37. Lindborg JA, Mack M, Zigmond RE. Neutrophils are critical for myelin removal in a peripheral nerve injury model of Wallerian degeneration. J Neurosci. 2017;37(43):10258-10277.

38. Hegedus J, Putman CT, Tyreman N, Gordon T. Preferential motor unit loss in the SOD1 G93A transgenic mouse model of amyotrophic lateral sclerosis. J Physiol (Lond). 2008;586(14):3337-3351.

39. Chen R, et al. Mast cells play a key role in neutrophil recruitment in experimental bullous pemphigoid. J Clin Invest. 2001;108(8):1151-1158.

40. Wezel A, et al. Mast cells mediate neutrophil recruitment during atherosclerotic plaque progression. Atherosclerosis. 2015;241(2):289-296.

41. Gorospe JR, Tharp M, Demitsu T, Hoffman EP. Dystrophin-deficient myofibers are vulnerable to mast cell granule-induced necrosis. Neuromuscul Disord. 1994;4(4):325-333.

42. Yokota M, et al. Roles of mast cells in the pathogenesis of inflammatory myopathy. Arthritis Res Ther. 2014;16(2):R72

43. Oskarsson B. Myopathy: five new things. Neurology. 2011;76(7 Suppl 2):S14-S19.

44. Krystel-Whittemore M, Dileepan KN, Wood JG. Mast cell: A multi-functional master cell. Front Immunol. 2015;6:620.

45. Lee SB, Kalluri R. Mechanistic connection between inflammation and fibrosis. Kidney Int Suppl. 2010;(119):S22-S26.

46. Levi-Schaffer F, et al. Nerve growth factor and eosinophils in inflamed juvenile conjunctival nevus. Invest Ophthalmol Vis Sci. 2002;43(6):1850-1856.

47. Gonzalez D, Contreras O, Rebolledo DL, Espinoza JP, van Zundert B, Brandan E. ALS skeletal muscle shows enhanced TGF- $\beta$ signaling, fibrosis and induction of fibro/adipogenic progenitor markers. PLoS One. 2017;12(5):e0177649.

48. Arecco N, et al. Elastase levels and activity are increased in dystrophic muscle and impair myoblast cell survival, proliferation and differentiation. Sci Rep. 2016;6:24708.

49. Takeuchi H, Kawasaki T, Shigematsu K, Kawamura K, Oka N. Neutrophil extracellular traps in neuropathy with anti-neutrophil cytoplasmic autoantibody-associated microscopic polyangiitis. Clin Rheumatol. 2017;36(4):913-917.

50. Dumont N, Bouchard P, Frenette J. Neutrophil-induced skeletal muscle damage: a calculated and controlled response following hindlimb unloading and reloading. Am J Physiol Regul Integr Comp Physiol. 2008;295(6):R1831-R1838.

51. Riley DA, Ilyina-Kakueva EI, Ellis S, Bain JL, Slocum GR, Sedlak FR. Skeletal muscle fiber, nerve, and blood vessel breakdown in space-flown rats. FASEB J. 1990;4(1):84-91.

52. Hodgetts S, Radley H, Davies M, Grounds MD. Reduced necrosis of dystrophic muscle by depletion of host neutrophils, or blocking TNFalpha function with Etanercept in mdx mice. Neuromuscul Disord. 2006;16(9-10):591-602.

53. Murdock BJ, et al. Increased ratio of circulating neutrophils to monocytes in amyotrophic lateral sclerosis. Neurol Neuroimmuno Neuroinflamm. 2016;3(4):e242.

54. Murdock BJ, Zhou T, Kashlan SR, Little RJ, Goutman SA, Feldman EL. Correlation of peripheral immunity with rapid amyotrophic lateral sclerosis progression. JAMA Neurol. 2017;74(12):1446-1454.

55. Tani K, et al. Chymase is a potent chemoattractant for human monocytes and neutrophils. J Leukoc Biol. 2000;67(4):585-589.

56. Zhuang X, Silverman AJ, Silver R. Brain mast cell degranulation regulates blood-brain barrier. J Neurobiol. 1996;31(4):393-403.

57. Sprague AH, Khalil RA. Inflammatory cytokines in vascular dysfunction and vascular disease. Biochem Pharmacol. 2009;78(6):539-552.

58. Theoharides TC, et al. Mast cells and inflammation. Biochim Biophys Acta. 2012;1822(1):21-33

59. Kano O, Beers DR, Henkel JS, Appel SH. Peripheral nerve inflammation in ALS mice: cause or consequence. Neurology. 2012;78(11):833-835.

60. Keizman D, et al. Low-grade systemic inflammation in patients with amyotrophic lateral sclerosis. Acta Neurol Scand. 2009;119(6):383-389.

61. Isaacs JD, Dean AF, Shaw CE, Al-Chalabi A, Mills KR, Leigh PN. Amyotrophic lateral sclerosis with sensory neuropathy: part of a multisystem disorder? J Neurol Neurosurg Psychiatry. 2007;78(7):750-753

62. Tian F, et al. Monitoring peripheral nerve degeneration in ALS by label-free stimulated Raman scattering imaging. Nat Commun 2016;7:13283. 